\title{
Distributed Risk-limiting Load Restoration for Wind Power Penetrated Bulk System
}

\author{
Zhao, Jin; Wang, Hongtao ; Wu, Qiuwei; Hatziargyriou, Nikos D.; Shen, Feifan
}

Published in:

IEEE Transactions on Power Systems

Link to article, DOI:

10.1109/TPWRS.2020.2973429

Publication date:

2020

Document Version

Peer reviewed version

Link back to DTU Orbit

Citation (APA):

Zhao, J., Wang, H., Wu, Q., Hatziargyriou, N. D., \& Shen, F. (2020). Distributed Risk-limiting Load Restoration for Wind Power Penetrated Bulk System. IEEE Transactions on Power Systems, 35(5), 3516 - 3528.

https://doi.org/10.1109/TPWRS.2020.2973429

\section{General rights}

Copyright and moral rights for the publications made accessible in the public portal are retained by the authors and/or other copyright owners and it is a condition of accessing publications that users recognise and abide by the legal requirements associated with these rights.

- Users may download and print one copy of any publication from the public portal for the purpose of private study or research.

- You may not further distribute the material or use it for any profit-making activity or commercial gain

- You may freely distribute the URL identifying the publication in the public portal 


\title{
Distributed Risk-limiting Load Restoration for Wind Power Penetrated Bulk System
}

\author{
Jin Zhao, Student Member, IEEE, Hongtao Wang, Senior Member, Qiuwei Wu, Senior Member, IEEE, \\ Nikos D. Hatziargyriou, Fellow, IEEE and Feifan Shen, Student Member, IEEE
}

\begin{abstract}
This paper proposes a new distributed risk-limiting load restoration scheme for wind power penetrated interconnected bulk systems. The conditional value-at-risk (CVaR) is used as the risk-limiting index. The projection function based alternating direction method of multipliers algorithm (P-ADMM) is used to decouple the CVaR integrated load restoration model into a distributed form. With the proposed algorithm, the risk-limiting constraints are decomposed and the complex non-convex load restoration model with the $\mathrm{CVaR}$ is transformed into small-scale scenario-based QP problems. The distributed risk-limiting load restoration scheme respects the individual decision-making of regional grids and reduces the security violation risk of the interconnected bulk system under uncertain conditions. The CVaR integrated P-ADMM method can efficiently reduce the computation complexity of the load restoration optimization for the uncertain bulk system without loss of optimality. The effectiveness of the proposed method was validated using the IEEE-118 bus system connected with ten IEEE-33 bus systems.
\end{abstract}

Index Terms-Alternating direction method of multipliers, power system load restoration, risk-limiting dispatch, wind power integration.

\section{NOMENCLATURE}

\section{A. Sets}

$N_{\mathrm{TS}} \quad$ Set of subsystems at the transmission level

$N_{\text {DS }} \quad$ Set of subsystems at the distribution level

$N_{\text {TL,TS } i}$ Set of loads in the $i$ th transmission system (TS)

$N_{\text {DL,DS } i}$ Set of loads in the $i$ th distribution system (DS)

$N_{\mathrm{G}, \mathrm{TS} i} \quad$ Set of generators in the $i$ th TS

$L_{\mathrm{T}, \mathrm{B}} \quad$ Set of boundary lines between TSs

$L_{\mathrm{DT}, \mathrm{B}} \quad$ Set of boundary lines between TSs and DSs

$N_{\text {RE,TS } i}$ Set of wind farms in the $i$ th TS

$N_{\mathrm{T}, \mathrm{TS} i} \quad$ Set of boundary nodes between TSs

$N_{\text {TD,TS } i}$ Set of boundary nodes between the $i$ th TS and DSs

$L_{\mathrm{TS} i} \quad$ Set of transmission lines in the $i$ th TS

$N_{\text {DT,DS } i}$ Set of boundary nodes between the $i$ th DS and TS

$N_{\text {DG,DS } i}$ Set of DGs in the $i$ th DS

$L_{\mathrm{DS} i} \quad$ Set of distribution lines in the $i$ th DS

This work was supported by the Science and Technology Foundation of SGCC (Research on Key Sub-station Control Technology for Rapid Recovery of AC/DC Hybrid Power Grid) (SGSDDK00KJJS1800084), (Corresponding author: Hongtao Wang, Qiuwei Wu).

J. Zhao and H. Wang are with the Key Laboratory of Power System Intelligent Dispatch and Control of Ministry of Education, Shandong University, Jinan 250000, China. (e-mail: jinzhao_sdu@mail.sdu.edu.cn; whtwhm@sdu. edu.cn).

Q. Wu and F. Shen are with the Center for Electric Power and Energy, Department of Electrical Engineering, Technical University of Denmark, Kgs. Lyngby, DK 2800 (e-mail: qw@elektro.dtu.dk; fshen@elektro.dtu.dk). N. D. Hatziargyriou is with the National Technical University of Athens, Athens 15773, Greece (e-mail: nh@power.ece.ntua.gr).

\section{B. Parameters}

$\boldsymbol{p}_{\mathrm{TL}}, \boldsymbol{p}_{\mathrm{DL}}$ active power of TS, DS loads

$$
\begin{aligned}
& {\left[\boldsymbol{p}_{\mathrm{TL}, \mathrm{TS}_{1}}, \ldots, \boldsymbol{p}_{\mathrm{TL}_{\mathrm{TS}}}, \ldots\right] \mathrm{TS}_{i} \in N_{\mathrm{TS}}} \\
& {\left[\boldsymbol{p}_{\mathrm{DL}, \mathrm{DS}}, \ldots, \boldsymbol{p}_{\mathrm{DL}, \mathrm{DS}_{i}}, \ldots\right] \mathrm{DS}_{i} \in N_{\mathrm{DS}}}
\end{aligned}
$$

$\boldsymbol{p}_{\mathrm{TL}, \mathrm{TS} i}, \boldsymbol{p}_{\mathrm{DL}, \mathrm{DS} i}$

Active power of loads of the $i$ th TS and $i$ th DS

$$
\begin{gathered}
{\left[p_{\mathrm{TL}, \mathrm{TS}_{i}, 1}, \ldots, p_{\mathrm{TL}, \mathrm{TS}_{i}, j}, \ldots\right] j \in N_{\mathrm{TL}, \mathrm{TS}_{i}}} \\
{\left[p_{\mathrm{DL}, \mathrm{DS}_{i}, 1}, \ldots, p_{\mathrm{DL}, \mathrm{DS}_{i}, j}, \ldots\right] j \in N_{\mathrm{DL}, \mathrm{DS}_{i}}}
\end{gathered}
$$

$p_{\mathrm{TL}, \mathrm{TS} i, j}, p_{\mathrm{DL}, \mathrm{DS} i, j}$

Active power of the $j$ th load in the $i$ th TS and $i$ th DS

$\boldsymbol{c}_{\mathrm{TL}}, \boldsymbol{c}_{\mathrm{DL}}$ Weighting coefficients of loads

$$
\begin{aligned}
& \operatorname{diag}\left(\boldsymbol{c}_{\mathrm{TL}, \mathrm{TS}_{1}}, \ldots, \boldsymbol{c}_{\mathrm{TL}, \mathrm{TS}_{i}}, \ldots\right) \mathrm{TS}_{i} \in N_{\mathrm{TS}} \\
& \operatorname{diag}\left(\boldsymbol{c}_{\mathrm{DL}, \mathrm{DS}_{1}}, \ldots, \boldsymbol{c}_{\mathrm{DL}, \mathrm{DS}_{i}}, \ldots\right) \mathrm{DS}_{i} \in N_{\mathrm{DS}}
\end{aligned}
$$

$\boldsymbol{c}_{\mathrm{TL}, \mathrm{TS} i}, \boldsymbol{c}_{\mathrm{DL}, \mathrm{DS} i}$

Weighting coefficients of loads in the $i$ th TS and $i$ th DS

$\operatorname{diag}\left(c_{\mathrm{TL}, \mathrm{TS}_{i}, 1}, \ldots, c_{\mathrm{TL}, \mathrm{TS}_{i}, j}, \ldots\right) j \in N_{\mathrm{TL} \mathrm{TS}_{i}}$

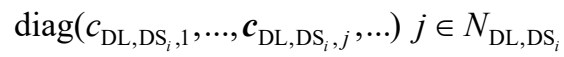

$c_{\mathrm{TL}, \mathrm{TS} i, j}, c_{\mathrm{DL}, \mathrm{DS} i, j}$

Weighting coefficients of the $j$ th load in the $i$ th TS and $i$ th DS

$\mathrm{R}_{\text {ind }}, \mathrm{R}_{\text {ind,TS } i}$ Risk indices for the whole system and $i$ th TS

$p_{\mathrm{G}, \mathrm{TS} i j}^{0}, p_{\mathrm{G}, \mathrm{TS} i, j, m i n}, p_{\mathrm{G}, \mathrm{TS} i j, \mathrm{max}}$

Initial, minimum, maximum active output of the $j$ th generator in the $i$ th TS

$p_{\mathrm{RE}, \mathrm{TS} i j} \quad$ Rated active power of $j$ th wind farm in the $i$ th TS

$r_{j} \quad$ Ramping rate of the $j$ th generator

$S_{n m, \max } \quad$ Apparent power limit of TS line $n m$

$h \quad$ The number of hyperplanes in the cosine polyhedron

$d \quad$ Distance between tangent points

$p_{\text {DG,DSi,n }} \quad$ Rated active power of DG at node $n$ in the $i$ th DS

$r_{n}, x_{n} \quad$ Resistance, reactance of DS line whose parent node is $n$

$L_{n, \max } \quad$ Apparent power limit of DS line whose parent node is $n$

$\Delta f_{\max } \quad$ Maximum frequency deviation

$s_{\mathrm{G}, \mathrm{TS}_{i}, j}, \varepsilon_{\mathrm{G}_{\mathrm{TT}}, j}$

Capacity, frequency response rate of the $j$ th generator in the $i$ th TS

$x_{\mathrm{TL}, \mathrm{TS} i}^{0}, n, x_{\mathrm{DL}, \mathrm{DS}_{i}, n}^{0}, x_{\mathrm{RE}, \mathrm{TS}_{i}, n}^{\mathrm{c}, 0}, x_{\mathrm{DG}, \mathrm{DS}}^{\mathrm{c}, 0}, n$

Initial decisions for TS load pickup, DS load pickup, wind farm output, DG output at node $n$ 
$\beta \quad$ Confidence level for the uncertain condition

$k \quad$ Iteration number

$K \quad$ Sampling number

$\rho \quad$ ADMM adjustable parameter

$\sigma_{1}, \sigma_{2} \quad$ Primal residual, dual residual convergence threshold

C. Variables

$\boldsymbol{x}_{\mathrm{TL}}, \boldsymbol{x}_{\mathrm{DL}} \quad \mathrm{TS}, \mathrm{DS}$ load pickup binary variables

$\left[\boldsymbol{x}_{\mathrm{TL}, \mathrm{TS}_{1}}, \ldots, \boldsymbol{x}_{\mathrm{TL}, \mathrm{TS}_{i}}, \ldots\right] \mathrm{TS}_{i} \in N_{\mathrm{TS}}$

$\left[\boldsymbol{x}_{\mathrm{DL}, \mathrm{DS}_{1}}, \ldots, \boldsymbol{x}_{\mathrm{DL}, \mathrm{DS}_{i}}, \ldots\right] \mathrm{DS}_{i} \in N_{\mathrm{DS}}$

$\boldsymbol{x}_{\mathrm{TL}, \mathrm{TS} i}, \boldsymbol{x}_{\mathrm{DL}, \mathrm{DS} i}$

Load pickup variables in the $i$ th TS and $i$ th DS

$\left[x_{\mathrm{TL} \mathrm{TS}_{i}, 1}, \ldots, x_{\mathrm{TL}, \mathrm{TS}_{i}, j}, \ldots\right] j \in N_{\mathrm{TL}^{\mathrm{TS}}{ }_{i}}$

$\left[x_{\mathrm{DL}, \mathrm{DS}_{i}, 1}, \ldots, x_{\mathrm{DL}, \mathrm{DS}}, j, \ldots\right] j \in N_{\mathrm{DL}, \mathrm{DS}_{i}}$

$x_{\mathrm{TL}, \mathrm{TS} i}, j, x_{\mathrm{DL}, \mathrm{DS} i, j}$

Load pickup variable of the $j$ th load in the $i$ th TS and $i$ th DS

$\boldsymbol{p}_{\mathrm{G}}, \boldsymbol{p}_{\mathrm{G}, \mathrm{TS} i}$ Generator output variables of the whole system and $i$ th TS

$$
\begin{aligned}
& {\left[\boldsymbol{p}_{\mathrm{G}, \mathrm{TS}_{1}}, \ldots, \boldsymbol{p}_{\mathrm{G}, \mathrm{TS}_{i}}, \ldots\right] \mathrm{TS}_{i} \in N_{\mathrm{TS}}} \\
& {\left[p_{\mathrm{G}, \mathrm{TS}_{i}, 1}, \ldots, p_{\mathrm{G}, \mathrm{TS}}, j, \ldots\right] j \in N_{\mathrm{G}, \mathrm{TS} i}}
\end{aligned}
$$

$p_{\mathrm{G}, \mathrm{TS} i, j} \quad$ Generators output variable of the $j$ th generator in the $i$ th TS.

$\boldsymbol{p}_{\mathrm{RE}}, \boldsymbol{p}_{\mathrm{RE}, \mathrm{TS} i}$

RES output variables of the whole system and $i$ th TS

$$
\begin{aligned}
& {\left[\boldsymbol{p}_{\mathrm{RE}, \mathrm{TS}_{1}}, \ldots, \boldsymbol{p}_{\mathrm{RE}, \mathrm{TS}_{i}}, \ldots\right] \mathrm{TS}_{i} \in N_{\mathrm{TS}}} \\
& {\left[p_{\mathrm{RE}, \mathrm{TS}_{i}, 1}, \ldots, p_{\mathrm{RE}, \mathrm{TS}_{i}}, j, \ldots\right] j \in N_{\mathrm{G}, \mathrm{TS} i}}
\end{aligned}
$$

$p_{\mathrm{RE}, \mathrm{TS} i, j}$ RES output variable of the $j$ th RES in the $i$ th TS.

$\boldsymbol{p}_{\mathrm{DG}}, \boldsymbol{p}_{\mathrm{DG}, \mathrm{DS} i}$

DG output variables of all the DSs and $i$ th DS

$$
\begin{aligned}
& {\left[\boldsymbol{p}_{\mathrm{DG}_{\mathrm{DS}}}, \ldots, \boldsymbol{p}_{\mathrm{DG}, \mathrm{DS}_{i}}, \ldots\right] \mathrm{DS}_{i} \in N_{\mathrm{DS}}} \\
& {\left[p_{\mathrm{DG}_{\mathrm{DS}}, 1}, \ldots, p_{\mathrm{DG}_{\mathrm{DS}}, j}, \ldots\right] j \in N_{\mathrm{DG}, \mathrm{DS} i}}
\end{aligned}
$$

$p_{\mathrm{DG}, \mathrm{DS} i, j}$ Output variable of the $j$ th DG in the $i$ th DS.

$\boldsymbol{Y} \quad$ Uncertain variables corresponding to variables in $\boldsymbol{D}$

D Risk-limiting constraint related variables of the whole system

$\boldsymbol{D}_{\mathrm{TS} i} \quad$ Risk-limiting constraint related variables of the $i$ th TS

$\boldsymbol{B}_{\mathrm{T}, \mathrm{TS} i j}, \boldsymbol{B}_{\mathrm{T}, \mathrm{TS} j i}$

Boundary variables between TSs for the $i$ th TS and $j$ th TS

$$
\begin{aligned}
& {\left[P_{\mathrm{B}, \mathrm{TS}_{i}, 1}, \ldots, P_{\mathrm{B}, \mathrm{TS}_{i}, n}, \ldots, \theta_{\mathrm{B}, \mathrm{TS}_{i}, 1}, \ldots, \theta_{\mathrm{B}, \mathrm{TS}_{i}, n}, \ldots\right]}
\end{aligned}
$$

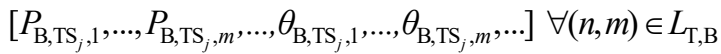

$P_{\mathrm{B}, \mathrm{TS} i, n}$ Boundary active power injected in node $n$ of the $i$ th TS $\theta_{\mathrm{B}, \mathrm{TS} i, n}$ Boundary phase angle at node $n$ of the $i$ th TS

$\boldsymbol{B}_{\mathrm{TD}, \mathrm{TS} i}, \boldsymbol{B}_{\mathrm{DT}, \mathrm{DS} j}$

Boundary variables between the TS and DS for the $i$ th TS and $j$ th DS

$\left[P_{\mathrm{B}, \mathrm{TS}_{i}, 1}, \ldots, P_{\mathrm{B}, \mathrm{TS}_{i}, n}, \ldots\right]$

$\left[P_{\mathrm{B}, \mathrm{DS}}, 1, \ldots, P_{\mathrm{B}, \mathrm{DS}}, m, \ldots\right] \forall(n, m) \in L_{\mathrm{DT}, \mathrm{B}}$

$P_{\mathrm{B}, \mathrm{DS} i, n}$ Boundary power injected in node $n$ of the $i$ th DS $R, R_{\mathrm{TS} i}$ Dynamic reserve for the whole system and $i$ th TS
$p_{\mathrm{G}, \mathrm{TS} i, j} \quad$ Output of the $j$ th generator in the $i$ th TS

$T \quad$ Load pickup time

$x_{\mathrm{RE}, \mathrm{TS}_{i, j}}^{\mathrm{c}}$ Continuous decision variable in $[0,1]$ for the output of the $j$ th wind farm in the $i$ th TS

$p_{\mathrm{G}, \mathrm{TS} i, n}, q_{\mathrm{G}, \mathrm{TS} i, n}$

Active, reactive output of generator at node $n$ in the $i$ th TS

$p_{n}, q_{n} \quad$ Active, reactive power injection into node $n$

$P_{n m}, Q_{n m}, S_{n m}$

Active, reactive, apparent power flow on TS line $\mathrm{nm}$

$V_{n} \quad$ Voltage magnitude at node $n$

$\delta_{n} \quad$ The difference between the target voltage and actual value at node $n$

$g_{n m}, b_{n m}, \theta_{n m}$

Conductance, susceptance, phase angle difference of TS line $n m$

$\cos ^{*} \theta_{n m}$ Approximation variable of $\cos \theta_{n m}$

$x_{\mathrm{DG}, \mathrm{DS} ;, n}^{\mathrm{c}}$ Continuous decision variable in $[0,1]$ for the DG output at node $n$ in the $i$ th DS

$p_{\mathrm{DS}, n}, q_{\mathrm{DS}, n}$ Active, reactive power injection into DS node $n$

$P_{\mathrm{DS}, n}, Q_{\mathrm{DS}, n}$ Active, reactive power flow from DS node $n$

$\Delta p_{\mathrm{TL}}, \Delta p_{\mathrm{DL}}, \Delta p_{\mathrm{RE}}, \Delta p_{\mathrm{DG}}$

Uncertain incremental changes of TS load amount, DS load amount, wind farm output, DG output

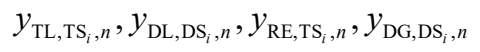

Uncertain variables of TS, DS load amount, TS wind farm output, DG output at node $n$

$\alpha_{\mathrm{TS} i} \quad$ Uncertainty threshold of the $i$ th TS $P_{\mathrm{B}, \mathrm{TS}_{i}, n}^{\mathrm{g}}, \theta_{\mathrm{B}, \mathrm{TS}_{i}, n}^{\mathrm{g}}$

Auxiliary variable for boundary active power, boundary phase angle at node $n$ between TSs

$P_{\mathrm{B}, \mathrm{TD}, n}^{\mathrm{g}} \quad$ Auxiliary variable for boundary active power at node $n$ between the TS and DS

$x_{\mathrm{TL}, \mathrm{TS}}^{\mathrm{g}, n}, x_{\mathrm{DL}, \mathrm{DS}_{i}, n}^{\mathrm{g}}$

Auxiliary variable for TS load pickup decision, DS load pickup decision at node $n$.

$\mu_{n}, \chi_{n}, \kappa_{n}, \omega_{n}, \eta_{n}, v_{n}$

Dual variables for decoupled boundary variables related to node $n$

\section{INTRODUCTION}

Tn recent years, power system blackouts are becoming more and more frequent because of extreme weather events with increasing intensity. Since a blackout incurs enormous social and economic losses, the efficient restoration after a system collapse becomes essential to enhance power system reliability and resilience. Power system restoration is an extremely complex and multi-faceted task which typically includes preparation, system restoration and load restoration stages [1]. The power system status assessment, faulted area isolation and units restarting are completed in the preparation stage. Then, the skeleton transmission paths are energized and islands are resynchronized in the system restoration stage. Finally, the remaining unserved loads are picked up in the load restoration stage. This paper focuses on the final stage of power system 
restoration, namely recovering the remaining load [1]. For successful accomplishment of the power system restoration, it is important to solve the 'when, which, how much' problem of load restoration [2], i.e., determine the load pickup time, location and amount.

This includes fast restoration decision-making [2]-[4] and solving optimization problems [5]-[7] generally carried out at the transmission system (TS) level by a centralized optimization algorithm. At the TS level, the load is regarded as load blocks with installed capacity the ratings of distribution transformers or feeders. This neglects the detailed operation characteristic of the distribution system (DS). Moreover, centralized optimization needs full information of the network, generator and load data of the whole bulk system consisting of several TSs and DSs. This information is normally not available in the interconnected bulk system, e.g., in the European systems transmission system operators (TSOs) and distribution system operators (DSOs) operate their networks mostly independently [8], while in the Chinese system, the central dispatch center is burdened by heavy data processing in order to operate the vertically managed interconnected bulk system [9].

Distributed optimization appears as an efficient way to coordinate the load restoration for the interconnected bulk system which contains several regional TSs and DSs [10]. Distributed methods allow parallel optimization of regional grids, and thus they can improve load restoration efficiency by considering details of the load blocks and decomposing the interconnected bulk system into several subsystems with smaller sizes and less variables [11]. The alternating direction method of multipliers (ADMM) [12] is a widely used algorithm to achieve distributed optimization in power system operation [13], [14] and control [11] problems. However, due to the difficulty of decomposing the security constraints and the presence of binary decision variables, it is difficult to use the standard ADMM in the load restoration models.

Moreover, renewable energy sources (RESs) have become an indispensable part of power supply in restoration process [2], [7], [15] imposing the need to take uncertainty into account. Risk limiting dispatch, with flexible uncertainty handling characteristic, is an effective way to deal with RES uncertainty [16]. Existing studies [17]-[19] however incorporate the risk related constraints in a coupled way, while distributed schemes require decoupling of risk constraints. Thus, further work should be done to integrate risk limitations into the distributed optimization method in order to provide a distributed risk-limiting load restoration scheme.

Methods such as robust optimization, chance-constrained programming and scenario-based stochastic programming can handle the uncertain condition. The robust optimization needs to define the uncertain boundary and chance-constrained programming requires the exact probabilistic distribution function. Scenario-based methods can obtain the optimal result according to scenarios directly. The stochastic programming method was employed in the restoration process in [6]. However, the computation efficiency is challenged when the number of scenarios is large. Another scenario-based method, the conditional value-at-risk $(\mathrm{CVaR})$ method, was employed in unit com- mitment [17], electricity market problems [19] and voltage control [20] to deal with all kinds of uncertainties. The CVaR method has the risk-tail representation ability and fast calculation characteristic even when the number of scenarios is large. Moreover, the CVaR method specially suits for the distributed optimization since its subadditivity can realize the decomposition of risk-limiting constraints.

In this paper, the CVaR index is integrated into a projection function based ADMM (P-ADMM) method to realize distributed risk-limiting load restoration in wind power penetrated bulk system with several independent TSs and DSs. Using the CVaR index [22], [23], the whole system risk-limiting constraint is transformed into distributed form and the P-ADMM method [24], [25] is employed to iteratively solve the nonconvex load restoration model. Since the P-ADMM method converts the binary variables into continuous variables by projection functions, the CVaR constraints are transformed into scenario-based linear constraints [22]. Finally, the distributed risk-limiting load restoration strategy can be obtained by iteratively solving quadratic programming $(\mathrm{QP})$ problem and applying projection functions. By incorporating the $\mathrm{CVaR}$ index into the P-ADMM method, the distributed risk-limiting load restoration optimization is efficiently solved.

The contributions of this paper are threefold. 1) It proposes a distributed risk-limiting load restoration scheme for wind power penetrated interconnected bulk system with several independent TSs and DSs. The bulk system load restoration problem is further studied considering several coupled TSs and DSs. The load blocks in the regional TSs are treated as independently operated by DSs. The concept of risk-limiting dispatch is extended to be the distributed risk-limiting one. 2) The standard ADMM method is extended to be the CVaR integrated P-ADMM algorithm which handles the uncertain condition and realizes the distributed risk-limiting load restoration. Without violating the whole system security, the CVaR method decouples risk-limiting constraints, which makes the P-ADMM method feasible, meanwhile, the P-ADMM method reduces the computation burden of $\mathrm{CVaR}$ based risk-limiting constraints. 3) The models are transformed into more computational tractable forms. The load restoration model of the large-scale interconnected system is transformed into several parallel subsystem based small-scale models, integer variables are dropped and risk-limiting constraints are linearized. The final models to be solved in the distributed risk-limiting scheme are small-scale QPs.

The rest of the paper is organized as follows. Section II introduces the coupled risk-limiting form of the TS-DS system load restoration. The detailed model of the distributed risklimiting load restoration is constructed in Section III. Section IV presents the iterative calculation process of the proposed models. Section V provides the case studies, followed by conclusions.

\section{FramewORK OF DistribUted RISK-LIMITING TS-DS LOAD RESTORATION SCHEME}

This section provides an overview of the distributed risk- 
limiting TS-DS load restoration. The whole system is divided into subsystems, and the centralized model is transformed into coupled sub-problem models corresponding to different TSOs and DSOs. The conditions for regional risk decomposition are listed next.

\section{A. Centralized risk-limiting load restoration model}

Load restoration is generally regarded as a step-by-step operation process realized by recursive optimization [2], [3], [5][7]. According to the generic risk-limiting optimization model in [17], the single-step centralized risk-limiting load restoration model of the interconnected bulk system with several interconnected TSs and DSs is formulated as (1)-(4).

$$
\begin{array}{r}
\operatorname{Max} \boldsymbol{p}_{\mathrm{TL}} \boldsymbol{c}_{\mathrm{TL}} \boldsymbol{x}_{\mathrm{TL}}^{\mathrm{T}}+\boldsymbol{p}_{\mathrm{DL}} \boldsymbol{c}_{\mathrm{DL}} \boldsymbol{x}_{\mathrm{DL}}^{\mathrm{T}} \\
\text { s.t. } \quad \boldsymbol{g}\left(\boldsymbol{x}_{\mathrm{TL}}, \boldsymbol{p}_{\mathrm{G}}, \boldsymbol{p}_{\mathrm{RE}}, \boldsymbol{x}_{\mathrm{DL}}, \boldsymbol{p}_{\mathrm{DG}}\right) \leq 0 \\
r(R \mid \boldsymbol{Y}) \leq \mathrm{R}_{\mathrm{ind}} \\
\left\{\boldsymbol{D}=f(\boldsymbol{Y}) \mid \boldsymbol{D}=\left[\boldsymbol{x}_{\mathrm{TL}}, \boldsymbol{p}_{\mathrm{G}}, \boldsymbol{x}_{\mathrm{DL}}\right]\right\}
\end{array}
$$

where $r($.$) is the risk index function of frequency security and$ $f($.) reflects the relationship between uncertain values and decision variables. The objective function (1) maximizes the load pickup amount considering their priorities. The first term means the total load pickup amount of the subsystem in the TS, while the second term is the total load pickup amount of DSs. Constraint (2) presents the operation constraints such as power supply limits and power flow and voltage constraints. Inequality (3) is the risk-limiting constraint. Constraint (4) links the decision variables with prediction information.

\section{B. Model division according to regional subsystems}

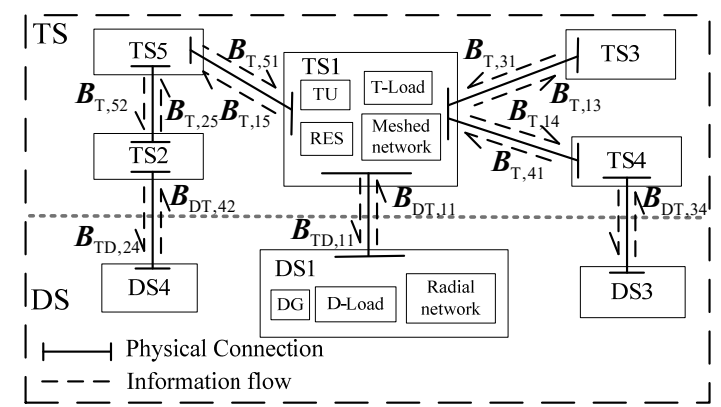

Fig. 1. The bulk system with several regional subsystems in TS and DS

As shown in Fig. 1, the interconnected bulk system consists of several TS and DS regional subsystems. At the TS level, a subsystem $\mathrm{TS}_{i}$ has a meshed structure and contains generators, large-scale RESs, e.g., wind farms, and loads. The subsystem DS $i$ at the DS level has a radial network, DGs and loads. Based on the structure of the TS-DS system, the original risklimiting load restoration model (1)-(4) can be equally rewritten as (5)-(10).

The objective function (5) is divided into the load amount of each TS/DS subsystem. Constraints (6) and (7) are the operation constraints of each TS and DS subsystem. The risk related constraints (8)-(9) are divided according to the TS subsystems. Constraint (10) maintains consensus of boundary variables on tie-lines between neighboring subsystems.

$\operatorname{Max} \sum_{\mathrm{TS}_{i} \in N_{\mathrm{TS}}}\left(\boldsymbol{p}_{\mathrm{TL}, \mathrm{TS}} \boldsymbol{c}_{\mathrm{TL}, \mathrm{TS} i} \boldsymbol{x}_{\mathrm{TL}, \mathrm{TS}}^{\mathrm{T}}\right)+\sum_{\mathrm{DS}_{i} \in N_{\mathrm{DS}}}\left(\boldsymbol{p}_{\mathrm{DL}, \mathrm{DS}} \boldsymbol{c}_{\mathrm{DL}, \mathrm{DS}} \boldsymbol{x}_{\mathrm{DL}, \mathrm{DS}}^{\mathrm{T}}\right)$

$$
\begin{aligned}
& \text { s.t. } g_{\mathrm{TS}_{i}}\left(\boldsymbol{x}_{\mathrm{TL}, \mathrm{TS}}, \boldsymbol{p}_{\mathrm{G}, \mathrm{TS} i}, \boldsymbol{p}_{\mathrm{RE}, \mathrm{TS}_{i}}, \boldsymbol{B}_{\mathrm{T}, \mathrm{TS} i}, \boldsymbol{B}_{\mathrm{TD}, \mathrm{TS}_{i}}\right) \leq 0 \\
& \forall\left(\mathrm{TS}_{i} \in N_{\mathrm{TS}}\right) \\
& g_{\mathrm{DS} i}\left(\boldsymbol{x}_{\mathrm{DL}, \mathrm{DS}}, \boldsymbol{p}_{\mathrm{DG}, \mathrm{DS}}, \boldsymbol{B}_{\mathrm{DT}, \mathrm{DS}}\right) \leq 0 \quad \forall\left(\mathrm{DS}_{i} \in N_{\mathrm{DS}}\right) \\
& r\left(\sum R_{\mathrm{TS}_{i}} \mid \boldsymbol{Y}\right) \leq \text { Risk } \quad \forall\left(\mathrm{TS}_{i} \in N_{\mathrm{TS}}\right) \\
& \left\{\boldsymbol{D}_{\mathrm{TS}_{i}}=f(\boldsymbol{Y}) \mid \boldsymbol{D}_{\mathrm{TS}_{i}}=\left[\boldsymbol{x}_{\mathrm{TL}, \mathrm{TS} i}, \boldsymbol{p}_{\mathrm{G}, \mathrm{TS}}, \boldsymbol{B}_{\mathrm{T}, \mathrm{TS} \mathrm{S}_{i j}}, \boldsymbol{B}_{\mathrm{TD}, \mathrm{TS} i}\right]\right\} \\
& \left\{\boldsymbol{B}_{\mathrm{T}, \mathrm{TS}}=\boldsymbol{B}_{\mathrm{T}, \mathrm{TS}_{j i} i}, \boldsymbol{B}_{\mathrm{DT}, \mathrm{DS}}=\boldsymbol{B}_{\mathrm{TD}, \mathrm{TS}}\right\}
\end{aligned}
$$

Although the risk-limiting model is divided by regional grid boundaries, boundary variables cannot be decoupled because of existing of constraints (8) and (10). If constraint (10) is relaxed, the risk-limiting model can be completely decomposed as long as the risk index satisfies (11). Then, (8) can be transformed into regional risk constraint (12) so that independent risk-limiting subsystems are formed. In order to satisfies (11) the CVaR is employed as the risk index and distributed risklimiting model is further formed, as explained in the next section.

$$
\begin{aligned}
& r\left(\sum R_{\mathrm{TS}_{i}} \mid \boldsymbol{Y}\right) \leq \sum r\left(R_{\mathrm{TS}_{i}} \mid \boldsymbol{Y}\right) \leq \mathrm{R}_{\text {ind }} \\
& \left\{\begin{array}{l}
r\left(R_{\mathrm{TS}_{i}} \mid \boldsymbol{Y}\right) \leq \mathrm{R}_{\text {ind, } \mathrm{TS}_{i}} \forall\left(\mathrm{TS}_{i} \in T S\right) \\
\sum \mathrm{R}_{\text {ind, } \mathrm{TS}_{i}}=\mathrm{R}_{\text {ind }}
\end{array}\right.
\end{aligned}
$$

\section{THE DisTRIBUTED RISK-LIMITING TS-DS LOAD RESTORATION MODEL}

The detailed distributed risk-limiting models are provided in this section. First, the centralized risk-limiting TS-DS system load restoration optimization model is presented. Then, the risk constraints are decomposed and transformed into scenario-based linear constraints. Finally, the distributed risklimiting models for TSOs and DSOs are described.

\section{A. Centralized risk-limiting load restoration model}

1) Objective function. The objective function (13) of the risk-limiting load restoration model is to maximize the whole system load pickup amount. The first term represents the sum of the load amount TS subsystems and the second term is the load amount of DS subsystems.

$$
\begin{aligned}
& \operatorname{Max} \sum_{\mathrm{TS}_{i} \in N_{\mathrm{TS}}} \sum_{j \in N_{\mathrm{TL}, \mathrm{TS}}}\left(c_{\mathrm{TL}, \mathrm{TS} i}, j p_{\mathrm{TL}, \mathrm{TS} i}, j x_{\mathrm{TL}, \mathrm{TS}_{i}, j}\right) \\
& +\sum_{\mathrm{DS}_{i} \in N_{\mathrm{DS}}} \sum_{j \in N_{\mathrm{DL}, \mathrm{DS}}}\left(c_{\mathrm{DL}, \mathrm{DS}}, j p_{\mathrm{DL}, \mathrm{DS} i, j} x_{\mathrm{DL}, \mathrm{DS}}, j\right)
\end{aligned}
$$

2) Constraints of the TS. The detailed constraints corresponding to (6) are shown in Appendix A. 1, which includes generator output limits (A-1)-(A-4) [5], [6], linearized AC power flow equations (A-5)-(A-12) [2], TS voltage constraints (A-13), linearized branch flow limits (A-14)-(A-17) and TS boundary power limits (A-18), for any subsystem at the TS level $\left(\mathrm{TS}_{i} \in N_{\mathrm{TS}}\right)$.

3) Constraints of the DS. The constraints corresponding to (7) are shown in Appendix A. 2, which contains linearized DisFlow constraints (A-19)-(A-23) [26], DS voltage limits (A24), linearized branch flow limits (A-25)-(A-28) and the DS boundary power limit (A-29), for any DS subsystem $\left(\mathrm{DS}_{i} \in\right.$ $N_{\text {DS) }}$. 
4) Centralized risk-limiting constraints. The bulk system frequency security is required in the restoration process [4]-[7], [27] and inequality (14) is a linear frequency limit considering the total capacity of restored generators and their frequency response rate. The frequency response rate is used to measure the largest frequency deviation in the transient process after system active power change [28].

$$
\left(\Delta p_{\mathrm{TL}}+\Delta p_{\mathrm{DL}}-\Delta p_{\mathrm{RE}}-\Delta p_{\mathrm{DG}}\right) \leq \Delta f_{\max } \sum_{\mathrm{TS}_{i} \in N_{\mathrm{TS}}} \sum_{j \in N_{\mathrm{GT}}, s_{\mathrm{G}}} s_{\mathrm{G}, \mathrm{TS}_{i}, j} / \varepsilon_{\mathrm{G}, \mathrm{TS}, j}
$$

Since the frequency security has to be maintained in the load restoration process, the risk of frequency security violation should be limited. The CVaR based risk-limiting constraints for (14) are (15)-(16) which ensures the conditional expected values of uncertain power demand should be within the governor response ability. In (16), the four terms in the right side calculate the uncertain incremental changes of TS load amount, DS load amount, RES output and DG output which corresponding to the left terms.

$$
\begin{aligned}
& \operatorname{CVaR}\left(\Delta p_{\mathrm{TL}}+\Delta p_{\mathrm{DL}}-\Delta p_{\mathrm{RE}}-\Delta p_{\mathrm{DG}}\right) \leq \Delta f_{\max } \sum_{\mathrm{TS}_{i} \in N_{\mathrm{TS}}} \sum_{j \in N_{\mathrm{G}, \mathrm{T}_{i}}} \frac{s_{\mathrm{G}, \mathrm{TS}_{i}, j}}{\varepsilon_{\mathrm{G}, \mathrm{TS}_{i}, j}} \\
& \Delta p_{\mathrm{TL}}+\Delta p_{\mathrm{DL}}-\Delta p_{\mathrm{RE}}-\Delta p_{\mathrm{DG}} \\
& =\sum_{\mathrm{TS}_{i} \in N_{\mathrm{TS}}} \sum_{n \in N_{\mathrm{TL}, \mathrm{TS}_{i}}}\left(x_{\mathrm{TL}, \mathrm{TS} i, n} y_{\mathrm{TL}, \mathrm{TS}_{i}, n}-x_{\mathrm{TL}, \mathrm{TS} i, n}^{0} p_{\mathrm{TL}_{\mathrm{TS}}, n}\right) \\
& +\sum_{\mathrm{DS}_{i} \in N_{\mathrm{DS}}} \sum_{n \in N_{\mathrm{DLL}} \mathrm{DS} i}\left(x_{\mathrm{DL}, \mathrm{DS}}, n y_{\mathrm{DL}, \mathrm{DS}}, n-x_{\mathrm{DL}, \mathrm{DS} i, n}^{0} p_{\mathrm{DL}, \mathrm{DS} i, n}\right) \\
& -\sum_{\mathrm{TS}_{i} \in N_{\mathrm{TS}}} \sum_{n \in N_{\mathrm{RE}, \mathrm{TS}_{i}}}\left(x_{\mathrm{RE}, \mathrm{TS}_{i}, n}^{\mathrm{c}} y_{\mathrm{RE}, \mathrm{TS} i, n}-x_{\mathrm{RE}, \mathrm{TS}_{i}, n}^{\mathrm{c}, 0} p_{\mathrm{RE}, \mathrm{TS}_{i}, n}\right)
\end{aligned}
$$

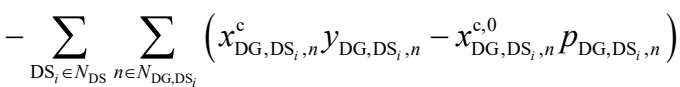

Equations (15)-(16) are consistent with (8)-(9) in the compact form of the risk-limiting model.

4) Coupling variables of TSs and DSs. The detailed constraints for (10) are,

$$
\begin{gathered}
P_{\mathrm{B}, \mathrm{TS}_{i}, n}=-P_{\mathrm{B}, \mathrm{TS}_{j}, m}\left(\forall(n, m) \in L_{\mathrm{T}, \mathrm{B}}\right) \\
\theta_{\mathrm{B}, \mathrm{TS}_{i}, n}=-\theta_{{\mathrm{B}, \mathrm{TS}_{j}, m}}\left(\forall(n, m) \in L_{\mathrm{T}, \mathrm{B}}\right) \\
P_{\mathrm{B}_{\mathrm{TS}}, n}=-P_{\mathrm{B}, \mathrm{DS}_{j}, m}\left(\forall(n, m) \in L_{\mathrm{DT}, \mathrm{B}}\right)
\end{gathered}
$$

where (17)-(18), and (19) ensure consensus of boundary variables on the tie-lines between TSs and TS and DSs, respectively.

\section{B. Distributed risk-limiting load restoration optimization}

According to the subadditivity of the CVaR [23], (20) corresponds to the inequality (11).

$$
\begin{aligned}
& \operatorname{CVaR}\left(\sum_{\mathrm{TS}_{i} \in N_{\mathrm{TS}}} f_{\mathrm{D}}\left(\boldsymbol{X}_{\mathrm{TS}_{i}}, \boldsymbol{Y}_{\mathrm{TS}_{i}}\right)\right) \leq \sum_{\mathrm{TS}_{i} \in N_{\mathrm{TS}}} \operatorname{CVaR}\left(f_{\mathrm{D}}\left(\boldsymbol{X}_{\mathrm{TS}_{i}}, \boldsymbol{Y}_{\mathrm{TS}_{i}}\right)\right) \\
& \leq \Delta f_{\max } \sum_{\mathrm{TS}_{i} \in N_{\mathrm{TS}}} \sum_{n, m \in N_{\mathrm{G}, \mathrm{T}_{i}}, n \neq m} \frac{s_{\mathrm{G}_{\mathrm{G}}, \mathrm{TS}_{i}, m}}{\varepsilon_{\mathrm{G}, \mathrm{TS}}, m}
\end{aligned}
$$

Thus, the risk constraints (15)-(16) for the whole system can be decoupled as (20)-(21) for each subsystem. The distributed risk constraints for the subsystems are (21)-(22) where the frequency security is maintained according to different TSs and the connected DSs. Equation (20) ensures the distributed transformation of risk constraints will not violate the frequency security requirement of the interconnected bulk system.

$$
\begin{aligned}
& \operatorname{CVaR}\left(f_{\mathrm{D}}\left(\boldsymbol{X}_{\mathrm{TS}_{i}}, \boldsymbol{Y}_{\mathrm{TS}_{i}}\right)\right) \leq \Delta f_{\max } \sum_{n, m \in N_{\mathrm{G}, \mathrm{ST}_{i}}, n \neq m} \frac{s_{\mathrm{G}, \mathrm{TS}_{i}, m}}{\varepsilon_{\mathrm{G}, \mathrm{TS}_{i}, m}} \\
& f_{\mathrm{D}}\left(\boldsymbol{X}_{\mathrm{TS}_{i}}, \boldsymbol{Y}_{\mathrm{TS}_{i}}\right)
\end{aligned}
$$

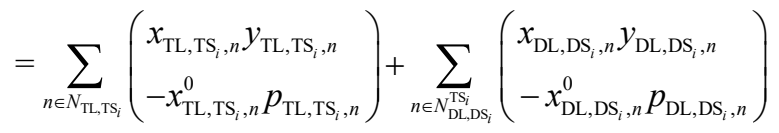

$$
\begin{aligned}
& -\sum_{n \in N_{\mathrm{RE}, \mathrm{TS}}}\left(\begin{array}{l}
x_{\mathrm{RE}, \mathrm{TS}_{i}, n}^{\mathrm{c}} y_{\mathrm{RE}, \mathrm{TS}_{i}, n} \\
-x_{\mathrm{RE}, \mathrm{TS}}^{\mathrm{c}, 0}, n \\
\mathrm{RE}_{\mathrm{RE}, \mathrm{TS}_{i}, n}
\end{array}\right)-\sum_{n \in N_{\mathrm{DS}, \mathrm{SS}}^{\mathrm{TS}}}\left(\begin{array}{l}
x_{\mathrm{DG}, \mathrm{DS}_{i}, n}^{\mathrm{c}} y_{\mathrm{DG}_{i}, \mathrm{DS}_{i}, n} \\
-x_{\mathrm{DG}, \mathrm{DS}_{i}, n}^{\mathrm{c}, 0} p_{\mathrm{DG}_{\mathrm{DS}}, n}
\end{array}\right)
\end{aligned}
$$

According to [22], the $\mathrm{CVaR}$ can be calculated using (23). Define $\alpha_{\mathrm{TS} i}$ as a threshold of function $f_{\mathrm{D}}$, the probability that $f_{\mathrm{D}}$ does not exceed the threshold $\alpha_{\mathrm{TS} i}$ is provided by (23).

$$
\Psi\left(\boldsymbol{X}_{\mathrm{TS}_{i}}, \alpha_{\mathrm{TS}_{i}}\right)=\int_{f_{\mathrm{D}}\left(\boldsymbol{X}_{\mathrm{TS}_{i}}, \boldsymbol{Y}_{\mathrm{TS}_{i}}\right) \leq a_{\mathrm{TS}_{i}}} \rho\left(\boldsymbol{Y}_{\mathrm{TS}_{i}}\right) d y
$$

where $\Psi\left(\boldsymbol{X}_{\mathrm{TS} i}, \alpha_{\mathrm{TS} i}\right)$ is the cumulative distribution function associated with $\boldsymbol{X}_{\mathrm{TS} i}$. The value-at-risk $(\mathrm{VaR})$ and $\mathrm{CVaR}$ values with any confidence level $\beta$ and decision variables $\boldsymbol{X}_{\mathrm{TS} i}$ can be obtained as (24)-(25).

$$
\begin{gathered}
\mathrm{VaR}: \alpha_{\mathrm{TS}_{i}}^{\beta}\left(\boldsymbol{X}_{\mathrm{TS}_{i}}\right)=\min \left\{\alpha_{\mathrm{TS}_{i}} \in R: \Psi\left(\boldsymbol{X}_{\mathrm{TS}_{i}}, \alpha_{\mathrm{TS}_{i}}\right) \geq \beta\right\} \\
\Phi_{\mathrm{D}}^{\beta}\left(\boldsymbol{X}_{\mathrm{TS}_{i}}\right)=\frac{1}{1-\beta} \int_{f_{\mathrm{D}}\left(\boldsymbol{X}_{\mathrm{TS}_{i}}, \boldsymbol{Y}_{\mathrm{TS}_{i}}\right) \geq \alpha_{\mathrm{TS}_{i}}^{\beta}\left(\boldsymbol{X}_{\mathrm{TS}_{i}}\right)} f_{\mathrm{D}}\left(\boldsymbol{X}_{\mathrm{TS}_{i}}, \boldsymbol{Y}_{\mathrm{TS}_{i}}\right) \rho\left(\boldsymbol{Y}_{\mathrm{TS}_{i}}\right) d y
\end{gathered}
$$

The VaR extracts the left endpoint of the corresponding nonempty interval of $\alpha_{\mathrm{TS} i}$ subject to $\Psi\left(\boldsymbol{X}_{\mathrm{TS} i}, \alpha_{\mathrm{TS} i}\right)=\beta$, and it is the threshold value of the uncertain variable related function $f_{\mathrm{D}}$ with the confidence level $\beta$. The CVaR is the conditional expected value of $f_{\mathrm{D}}$ that exceeds VaR. Since (23) is computation intractable, (26) is provided [22]. It satisfies (27), therefore, the $\mathrm{CVaR}$ value can be calculated by optimizing (26).

$$
\begin{array}{r}
F_{\mathrm{D}}^{\beta}\left(\boldsymbol{X}_{\mathrm{TS}_{i}}, \alpha_{\mathrm{TS}_{i}}\right)=\alpha_{\mathrm{TS}_{i}}+\frac{1}{1-\beta} \int_{y \in R^{n}}\left[f_{\mathrm{D}}\left(\boldsymbol{X}_{\mathrm{TS}_{i}}, \boldsymbol{Y}_{\mathrm{TS}_{i}}\right)-\alpha_{\mathrm{TS}_{i}}\right]^{+} \rho\left(\boldsymbol{Y}_{\mathrm{TS}_{i}}\right) d y \\
{[Z]^{+}=\max \{0, Z\}} \\
\min _{\left(\boldsymbol{X}_{\mathrm{TS}_{i}}, \alpha_{\mathrm{TS}_{i}}\right) \in X \times R} F_{\mathrm{D}}^{\beta}\left(\boldsymbol{X}_{\mathrm{TS}_{i}}, \alpha_{\mathrm{TS}_{i}}\right)=\min _{\boldsymbol{X}_{\mathrm{TS}_{i} \in X}} \Phi_{\mathrm{D}}^{\beta}\left(\boldsymbol{X}_{\mathrm{TS}_{i}}\right)
\end{array}
$$

To handle the integral part and the intractable analytical expression of $\rho(\boldsymbol{Y})$, the following approximation is used as (28) where $\boldsymbol{Y}_{\mathrm{TS} i}^{*}=\left[\boldsymbol{Y}_{\mathrm{TS} i, 1,}^{*}, \ldots, \boldsymbol{Y}_{\mathrm{TS} i, K}^{*}, \ldots\right]$ is a collection of uncertain variables sampling. Finally, (21) can be transformed into (29) [29].

$$
\begin{gathered}
F_{\mathrm{D}}^{\beta^{*}}\left(\boldsymbol{X}_{\mathrm{TS}_{i}}, \alpha_{\mathrm{TS}_{i}}\right)=\alpha_{\mathrm{TS}_{i}}+\frac{1}{\mathrm{M}(1-\beta)} \sum_{K=1}^{\mathrm{M}}\left[f_{\mathrm{D}}\left(\boldsymbol{X}_{\mathrm{TS}_{i}}, \boldsymbol{Y}_{\mathrm{TS}_{i}, k}^{*}\right)-\alpha_{\mathrm{TS}_{i}}\right]^{+} \\
F_{\mathrm{D}}^{\beta^{*}}\left(\boldsymbol{X}_{\mathrm{TS}_{i}}, \alpha_{\mathrm{TS}_{i}}\right) \leq \Delta f_{\max } \sum_{n, m \in N_{\mathrm{G}, \mathrm{TS}_{i}}, n \neq m} \frac{s_{\mathrm{G}_{\mathrm{TS}}, m}}{\varepsilon_{\mathrm{G}_{\mathrm{T}}, \mathrm{TS}_{i}, m}}
\end{gathered}
$$

With the transformation above, the distributed risk-limiting sub-problem for each TS subsystem is Model (30) and the one for each DS is Model (31). The cold load pickup phenomenon can be included using the linear models in [2]. In the distributed scheme, the whole system frequency security is maintained by the subsystem frequency limit. 


$$
\begin{aligned}
& \operatorname{Max}_{x_{\mathrm{TL}, \mathrm{TS} i}, j, x_{\mathrm{RE}, \mathrm{TS}_{i}, n}^{\mathrm{c}}, p_{\mathrm{G}, n}, P_{\mathrm{B}, \mathrm{TS}_{i}, n}, w_{\mathrm{TS}_{i}}} \sum_{j \in N_{\mathrm{TL}, \mathrm{TS}}}\left(c_{\mathrm{TL}, \mathrm{TS}_{i}, j} p_{\mathrm{TL}_{\mathrm{TS}}, j} x_{\mathrm{TL}, \mathrm{TS}_{i}, j}\right) \\
& \text { s.t. }(\mathrm{A}-1)-(\mathrm{A}-18),(17)-(19),(28)-(29)
\end{aligned}
$$

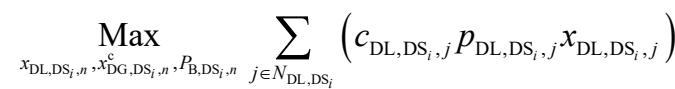

$$
\begin{aligned}
& \text { s.t. }(\mathrm{A}-19)-(\mathrm{A}-29),(19)
\end{aligned}
$$

Because of the existing of boundary consensus constraints (17)-(22), these regional models are still coupled. Besides, although the power flow related constraints have been linearized and risk constraints are converted to simpler forms, the risk constraints are still computation-intractable since the threshold $\alpha_{\mathrm{TS} i}$ is difficult to be calculated. The CVaR integrated P-ADMM method is developed in Section IV to solve the distributed risk-limiting Model (30) and (31).

\section{SOlution METHOD FOR THE DiSTRIBUTED RiSK-LIMITING LOAD RESTORATION OPTIMIZATION}

This section introduces the iterative calculation process to solve the distributed risk-limiting model. The P-ADMM method [24], [25] is used and the CVaR constraints are linearized. The distributed risk-limiting load restoration strategy can be obtained by iteratively solving small scale QP problems with projected binary variables.

\section{A. Sub-problem decoupling and integer variable processing}

First, the constraints for boundary variables (17)-(19) are relaxed by the augmented Lagrangian method to decouple the sub-problems. Thus, a set of auxiliary variables $\left(\boldsymbol{P}_{T S}^{\mathrm{g}}, \boldsymbol{\theta}_{T S}^{\mathrm{g}}, \boldsymbol{P}_{D S}^{\mathrm{g}}\right)$ and equality constraints (32)-(34) corresponding to the boundary constraints are introduced. Furthermore, note that the binary variables $\left(\boldsymbol{x}_{\mathrm{TL}}, \boldsymbol{x}_{\mathrm{DL}}\right)$ in Model (30) and (31) are treated as continuous, the auxiliary binary variables $\left(\boldsymbol{x}_{\mathrm{TL}}^{\mathrm{g}}, \boldsymbol{x}_{\mathrm{DL}}^{\mathrm{g}}\right)$ and two additional equalities (35)-(36) are introduced.

$$
\begin{aligned}
& P_{\mathrm{B}, \mathrm{TS}_{i}, n}=P_{\mathrm{B}, \mathrm{TS}_{i}, n}^{\mathrm{g}} \& P_{\mathrm{B}, \mathrm{TS}_{j}, m}=-P_{\mathrm{B}, \mathrm{TS}_{i}, n}^{\mathrm{g}}\left(\forall(n, m) \in L_{\mathrm{T}, \mathrm{B}}\right) \\
& \theta_{\mathrm{B}, \mathrm{TS}_{i}, n}=\theta_{\mathrm{B}, \mathrm{TS}_{i}, n}^{\mathrm{g}} \& \theta_{{\mathrm{B}, \mathrm{TS}_{j}, m}}=-\theta_{\mathrm{B}, \mathrm{TS}_{i}, n}^{\mathrm{g}}\left(\forall(n, m) \in L_{\mathrm{T}, \mathrm{B}}\right) \\
& P_{\mathrm{B}, \mathrm{TS}_{i}, n}=P_{\mathrm{B}, \mathrm{TD}, n}^{\mathrm{g}} \& P_{\mathrm{B}, \mathrm{DS}_{j}, m}=-P_{\mathrm{B}, \mathrm{TD}, n}^{\mathrm{g}}\left(\forall(n, m) \in L_{\mathrm{DT}, \mathrm{B}}\right) \\
& x_{\mathrm{TL}, \mathrm{TS}_{i}, n}=x_{\mathrm{TL}, \mathrm{TS}_{i}, n}^{\mathrm{g}}\left(n \in N_{\mathrm{TL}_{\mathrm{TS}} i}, x_{\mathrm{TL}, \mathrm{TS}_{i}, n} \in[0,1], x_{\mathrm{TL}, \mathrm{TS}_{i}, n}^{\mathrm{g}} \in\{0,1\}\right) \\
& x_{\mathrm{DL}, \mathrm{DS}_{i}, n}=x_{\mathrm{DL}, \mathrm{DS}_{i}, n}^{\mathrm{g}}\left(n \in N_{\mathrm{DL}, \mathrm{DS}_{i}}, x_{\mathrm{DL}, \mathrm{DS}_{i}, n} \in[0,1], x_{\mathrm{DL}^{\mathrm{g}} \mathrm{DS}_{i}, n}^{\mathrm{g}} \in\{0,1\}\right)
\end{aligned}
$$

According to the augmented Lagrangian method, the constraints of boundary variables and binary variables (17)-(19) can be decoupled by adding relaxed constraints (17)-(19) into the objective function. Therefore, the sub-problem models for TS subsystems and DS subsystems are transformed into (37) and (38) where $\mu, \chi, \kappa, \omega, \eta$ and $v$ are the dual variables of the corresponding relaxed constraints. Models (37) and (38) are distributed risk-limiting load restoration models for the TS and DS, respectively. In Model (37), the first term of the objective function represents the weighted load recovery amount of the $i$ th TS, while the other quadratic terms are augmented Lagrangian terms for (32), (33), the first equation in (34) and (35). The first term of the objective function in Model (38) denotes the weighted load recovery amount of the $i$ th DS, and the rest quadratic ones are augmented Lagrangian terms for the second equation in (34) and (36).

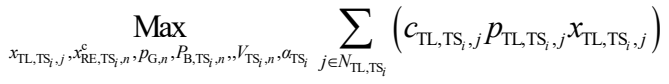

$$
\begin{aligned}
& -\frac{1}{2} \rho\left\{\sum _ { ( n , m ) \in L _ { \mathrm { T } , \mathrm { B } , \mathrm { TS } _ { i } } } \left(\left\|P_{\mathrm{B}, \mathrm{TS}_{i}, n}^{\mathrm{g}}-P_{\mathrm{B}, \mathrm{TS}_{i}, n}+\mu_{n}\right\|^{2}-\left\|\mu_{n}\right\|^{2}+\| P_{\mathrm{B}, \mathrm{TS}_{i}, m}^{\mathrm{g}}+P_{\mathrm{B}, \mathrm{TS}_{i}, m}\right.\right. \\
& +\mu_{m}\left\|^{2}-\right\| \mu_{m} \|^{2}+\sum_{(n, m) \in L_{\mathrm{T}, \mathrm{B}, \mathrm{T}_{i}}}\left(\left\|\theta_{\mathrm{B}, \mathrm{TS}_{i}, n}^{\mathrm{g}}-\theta_{\mathrm{B}, \mathrm{TS}_{i}, n}+\chi_{n}\right\|^{2}-\left\|\chi_{n}\right\|^{2}+\| \theta_{\mathrm{B}, \mathrm{TS}_{i}, m}^{\mathrm{g}}\right. \\
& \left.+\theta_{\mathrm{B}, \mathrm{TS}_{i}, m}+\chi_{m}\|-\| \chi_{m} \|^{2}\right)+\sum_{n \in N_{\mathrm{TD}, \mathrm{TS}}}\left(\left\|P_{\mathrm{B}, \mathrm{TD}_{i}, n}^{\mathrm{g}}-P_{\mathrm{B}, \mathrm{TD}, n}+\kappa_{n}\right\|^{2}-\left\|\kappa_{n}\right\|^{2}\right) \\
& \left.+\sum_{n \in N_{\mathrm{T}, \mathrm{TS}_{i}}}\left(\left\|x_{\mathrm{TL}^{\mathrm{g}}, \mathrm{TS}_{i}, n}-x_{\mathrm{TL}, \mathrm{TS}_{i}, n}+\omega_{n}\right\|^{2}-\left\|\omega_{n}\right\|^{2}\right)\right\} \\
& \text { s.t. }(\mathrm{A}-1)-(\mathrm{A}-4) \quad \text { generator output constraints } \\
& \text { (A-5)-(A-12) TS ACpower flow constraints } \\
& \text { (A-13)-(A-17) voltage/line flow constraints } \\
& \text { (A-18) TSboundary power constraint } \\
& \text { (28)-(29) frequencyconstraints } \\
& x_{\mathrm{TL}, \mathrm{TS}_{i}, n} \in[0,1], x_{\mathrm{TL}, \mathrm{TS}_{i}, n}^{\mathrm{g}} \in\{0,1\}
\end{aligned}
$$

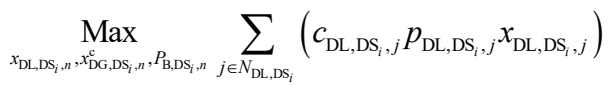

$$
\begin{aligned}
& -\frac{1}{2} \rho\left\{\sum _ { n \in N _ { \mathrm { DT } , \mathrm { DS } } } \left(\left\|P_{\mathrm{B}, \mathrm{TD}, n}^{\mathrm{g}}+P_{\mathrm{B}, \mathrm{DS}_{j}, n}+\eta_{n}\right\|^{2}-\left\|\eta_{n}\right\|^{2}\right.\right. \\
& \left.+\sum_{n \in N_{\mathrm{DL}, \mathrm{DS}}}\left(\left\|x_{\mathrm{DL}, \mathrm{DS}_{i}, n}^{\mathrm{g}}-x_{\mathrm{DL}, \mathrm{DS}_{i}, n}+v_{n}\right\|^{2}-\left\|v_{n}\right\|^{2}\right)\right\} \\
& \text { s.t. }(\mathrm{A}-19)-(\mathrm{A}-23) \quad \text { DisFlowconstraints } \\
& (\mathrm{A}-24)-(\mathrm{A}-28) \quad \text { voltage/line flowconstraints } \\
& \text { (A-29) DS boundary power constraints } \\
& x_{\mathrm{DL}, \mathrm{DS}_{i}, n} \in[0,1], x_{\mathrm{DL}, \mathrm{DS}_{i}, n}^{\mathrm{g}} \in\{0,1\}
\end{aligned}
$$

where auxiliary variables and binary variables are only included in the objective functions of the augmented Lagrangian.

\section{B. Iterative calculation process of CVaR integrated P-ADMM algorithm}

In the P-ADMM algorithm, the optimization over primal variables $(\mathbf{X}, \mathbf{Z})$ is separated in two optimization models. The primal variables and dual variables $(\boldsymbol{\Lambda})$ are updated in an iterative process which is listed as four steps below.

$$
\left\{\begin{aligned}
\mathbf{X}= & {\left[\boldsymbol{x}_{\mathrm{TL}}, \boldsymbol{x}_{\mathrm{RE}}^{\mathrm{c}}, \boldsymbol{p}_{\mathrm{G}}, \boldsymbol{P}_{\mathrm{B}, \mathrm{TS}}, \boldsymbol{\theta}_{\mathrm{B}, \mathrm{TS}}, \boldsymbol{V}_{\mathrm{TS}},\right.} \\
& \left.\boldsymbol{\alpha}, \boldsymbol{x}_{\mathrm{DL}}, \boldsymbol{x}_{\mathrm{DG}}^{\mathrm{c}}, \boldsymbol{P}_{\mathrm{B}, \mathrm{DS}}, \boldsymbol{V}_{\mathrm{DS}}\right] ; \\
\mathbf{Z}= & {\left[\boldsymbol{P}_{\mathrm{B}, \mathrm{TS}}^{\mathrm{g}}, \boldsymbol{\theta}_{\mathrm{B}, \mathrm{TS}}^{\mathrm{g}}, \boldsymbol{P}_{\mathrm{B}, \mathrm{TD}}^{\mathrm{g}}, \boldsymbol{x}_{\mathrm{TL}}^{\mathrm{g}}, \boldsymbol{x}_{\mathrm{DL}}^{\mathrm{g}}\right] ; } \\
\boldsymbol{\Lambda}= & {[\boldsymbol{\mu}, \boldsymbol{\chi}, \boldsymbol{\kappa}, \boldsymbol{\omega}, \boldsymbol{\eta}, \boldsymbol{v}] ; }
\end{aligned}\right.
$$

1) Stepl (Solving models with primal variables): At the $k$ th iteration, the optimization models for TS and DS subproblems are given by (40) and (41), in which primal variables $\mathbf{X}$ are optimized, and primal variables $\mathbf{Z}$ and dual variables $\boldsymbol{\Lambda}$ are considered as parameters $\mathbf{Z}^{k-1^{*}}$ and $\boldsymbol{\Lambda}^{k-1^{*}}$ obtained at the $(k$ 1)th iteration.

With all the binary variables in primal variables fixed, the CVaR based risk index (28) becomes convex [29] and the risk constraints (28)-(29) can be replaced by a linear function and an additional set of linear constraints [22]. Define auxiliary 
variable $u_{\mathrm{TS}, K}^{k}$, and $u_{\mathrm{TS}, K}^{k}=\left[f_{\mathrm{D}}\left(\boldsymbol{X}_{\mathrm{TS},}^{k}, \boldsymbol{Y}_{\mathrm{TS}, K}^{*}\right)-\alpha_{\mathrm{TS}}^{k}\right]^{+}$. Risk-limiting constraints (28)-(29) can be replaced by the scenario-based linear constraints (42)-(43). To completely decouple the TS and connected DSs, the DS variables $\boldsymbol{x}_{\mathrm{DL}}$ and $\boldsymbol{x}_{\mathrm{DG}}^{\mathrm{c}}$ in $f_{\mathrm{D}}()$ are fixed with the values obtained at the $(k-1)$ th iteration.

$$
\begin{aligned}
& \underset{\boldsymbol{X}_{\mathrm{TS}_{i}}^{k}}{\operatorname{Max}} \sum_{j \in N_{\mathrm{TL}, \mathrm{TS}_{i}}}\left(c_{\mathrm{TL}, \mathrm{TS}_{i}, j} p_{\mathrm{TL}_{\mathrm{TS}}, j} x_{\mathrm{TL}, \mathrm{TS}_{i}, j}^{k}\right) \\
& -\frac{1}{2} \rho\left\{\sum _ { ( n , m ) \in L _ { \mathrm { T } , \mathrm { B } , \mathrm { S } _ { i } } } \left(\left\|P_{\mathrm{B}, \mathrm{TS}_{i}, n}^{k}-P_{\mathrm{B}, \mathrm{TS}_{i}, n}^{\mathrm{g}, \mathrm{I}^{*}}+\mu_{n}^{k-1^{*}}\right\|^{2}-\left\|\mu_{n}^{k-1^{*}}\right\|^{2}+\| P_{\mathrm{B}, \mathrm{TS}_{i}, m}^{k}+P_{\mathrm{B}, \mathrm{TS}_{i}, m}^{\mathrm{g}, k \mathbb{*}^{*}}\right.\right. \\
& +\mu_{m}^{k-1 * 1^{*}}\left\|^{2}-\right\| \mu_{m}^{k-1^{*}} \|^{2}+\sum_{(n, m) \in L_{\mathrm{T}, \mathrm{BTS}}}\left(\left\|\theta_{\mathrm{B}, \mathrm{TS}, n}^{k}-\theta_{\mathrm{B}, \mathrm{s} \mathrm{TS}_{i}, n}^{\mathrm{g}, k-1^{*}}+\chi_{n}^{k-1^{*}}\right\|^{2}-\left\|\chi_{n}^{k-1^{*}}\right\|^{2}+\| \theta_{\mathrm{B}, \mathrm{TS}, m}^{k}\right. \\
& \left.+\theta_{\mathrm{B}, \mathrm{TS}_{i}, m}^{g, k-1^{*}}+\chi_{m}^{k-1^{*}}\|-\| \chi_{m}^{k-1^{*}} \|^{2}\right)+\sum_{n \in N_{\mathrm{TD}, \mathrm{TS}}}\left(\left\|P_{\mathrm{B}, \mathrm{TS}_{i}, n}^{k}-P_{\mathrm{B}, \mathrm{TD}, n}^{g, k-1^{*}}+\kappa_{n}^{k-1 * *^{*}}\right\|^{2}-\left\|\kappa_{n}^{k-1^{*}}\right\|^{2}\right) \\
& \left.+\sum_{n \in N_{\mathrm{T}, \mathrm{TS}_{i}}}\left(\left\|x_{\mathrm{TL}, \mathrm{TS}_{i}, n}^{k}-x_{\mathrm{TL}, \mathrm{TS}_{i}, n}^{\mathrm{g}, \mathrm{l}^{*}}+\omega_{n}^{k-1^{*}}\right\|^{2}-\| \omega_{n}^{k-1^{*} \|^{2}}\right)\right\} \\
& \text { s.t. }(\mathrm{A}-1)-(\mathrm{A}-18),(28)-(29), x_{\mathrm{TL}, \mathrm{TS}, n}^{k} \in[0,1]
\end{aligned}
$$$$
\operatorname{Max}_{X_{\mathrm{DS} i}} \sum_{j \in N_{\mathrm{DL}, \mathrm{DS}}}\left(c_{\mathrm{DL}, \mathrm{DS} i}, j p_{\mathrm{DL}, \mathrm{DS}}, j x_{\mathrm{DL}, \mathrm{DS} i}^{k}, j\right)-\frac{1}{2} \rho\left\{\sum _ { n \in N _ { \mathrm { DT } , \mathrm { DS } } } \left(\| P_{\mathrm{B}, \mathrm{DS}}^{k}, n+P_{\mathrm{B}, \mathrm{TD}, n}^{g, k *^{*}}\right.\right.
$$$$
\left.\left.+\eta_{n}^{k-1^{*}}\left\|^{2}-\right\| \eta_{n}^{k-1^{*}} \|^{2}\right)+\sum_{n \in N_{\mathrm{DL}, \mathrm{DS}}}\left(\left\|x_{\mathrm{DL}, \mathrm{DS}}^{k}, n-x_{\mathrm{DL}, \mathrm{DS}}^{g, n}, n+v_{n}^{k-1^{*}}\right\|^{2}-\left\|v_{n}^{k-1^{*}}\right\|^{2}\right)\right\}
$$$$
\text { s.t. }(\mathrm{A}-19)-(\mathrm{A}-29), x_{\mathrm{DL}, \mathrm{DS} i, n}^{k} \in[0,1]
$$$$
\alpha_{\mathrm{TS}_{i}}^{k}+\frac{1}{\mathrm{M}(1-\beta)} \sum_{K=1}^{M} u_{\mathrm{TS}_{i}, K}^{k} \leq \Delta f_{\max } \sum_{n, m \in N_{\mathrm{G}, \mathrm{TS}}, n \neq m} \frac{s_{\mathrm{G}, \mathrm{TS}_{i}, m}}{\varepsilon_{\mathrm{G}, \mathrm{TS}_{i}, m}}
$$$$
u_{\mathrm{TS}_{i}, K}^{k} \geq f_{\mathrm{D}}\left(\boldsymbol{X}_{\mathrm{TS}_{i}}^{k}, \boldsymbol{Y}_{\mathrm{TS}_{i}, K}^{*}\right)-\alpha_{\mathrm{TS}_{i}}^{k}, u_{\mathrm{TS}_{i}, K}^{k} \geq 0 K=1, \ldots, \mathrm{M}
$$

Till now, the TS and DS subsystem models are formulated as QP problem and can be optimized in a distributed manner.

2) Step2 (Solving models with primal variables): With the obtained values in $\mathbf{X}^{k^{*}}$ and $\boldsymbol{\Lambda}^{k-1^{*}}$, (44) and (45) for each TS and DS are calculated to obtain the values of primal variables in $\mathbf{Z}$ at the $k$ th iteration.

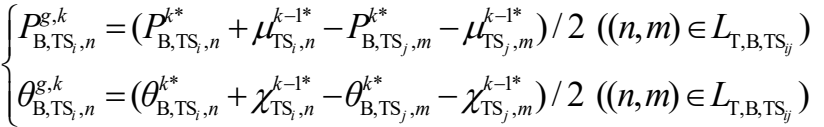

$$
\begin{aligned}
& P_{\mathrm{B}, \mathrm{TD}, n}^{\mathrm{g}, k}=\left(P_{\mathrm{B}, \mathrm{TS}_{i}, n}^{k^{*}}+\kappa_{\mathrm{TS}_{i}, n}^{k-1^{*}}-P_{\mathrm{B}, \mathrm{DS}_{j}, m}^{k^{*}}-\eta_{\mathrm{DS}_{j}, m}^{k-1^{*}}\right) / 2\left((n, m) \in L_{\mathrm{TD}, \mathrm{B}}\right)
\end{aligned}
$$

The binary variables are updated using the projection functions (46).

$$
\left\{\begin{array}{l}
x_{\mathrm{TL}, \mathrm{TS}_{i}, n}^{\mathrm{g}, k}=\Pi_{x}\left(x_{\mathrm{TL}, \mathrm{TS}_{i}, n}^{k^{*}}+\omega_{n}^{k-1^{*}}\right)\left(n \in N_{\mathrm{TL}, \mathrm{TS}_{i}}\right) \\
x_{\mathrm{DL}, \mathrm{DS}_{i}, n}^{\mathrm{g}, k}=\Pi_{x}\left(x_{\mathrm{DL}, \mathrm{DS}_{i}, n}^{k^{*}}+v_{n}^{k-1^{*}}\right)\left(n \in N_{\mathrm{DL}, \mathrm{DS}}\right)
\end{array}\right.
$$

where $\Pi$ represents the projection onto $x$, which means $\Pi$ round the argument to its nearest binary value. The models in this step are also calculated according to subsystems in a distributed manner.

3) Step3 (Dual variable update): The dual variables are updated according to (48) where $\mathbf{X}^{\mathrm{E}, k^{*}}$ contains the boundary variables between subsystems and load pickup variables.

$$
\begin{aligned}
\mathbf{X}^{\mathrm{E}, k^{*}}= & {\left[\boldsymbol{P}_{\mathrm{B}, \mathrm{TS}}^{k^{*}}, \boldsymbol{\theta}_{\mathrm{B}, \mathrm{TS}}^{k^{*}}, \boldsymbol{P}_{\mathrm{B}, \mathrm{DS}}^{k^{*}}, \boldsymbol{x}_{\mathrm{TL}}^{k^{*}}, \boldsymbol{x}_{\mathrm{DL}}^{k^{*}}\right] } \\
& \boldsymbol{\Lambda}^{k}=\boldsymbol{\Lambda}^{k-1^{*}}+\left(\mathbf{X}^{\mathrm{E}, k^{*}}-\mathbf{Z}^{k^{*}}\right)
\end{aligned}
$$

4) Step4 (Stopping criterion): Go back to Step1 if (49), which denotes the primal and dual residuals are lower than a specified tolerance, is not satisfied. Otherwise, the iteration process stops. If there are lots of binary variables, corresponding to small load amount in DSs, the primal residual for binary variables can be relaxed to avoid too many iterations.

$$
\left\|\mathbf{X}^{\mathrm{E}, k^{*}}-\mathbf{Z}^{k^{*}}\right\| \leq \sigma_{1} \wedge\left\|\mathbf{X}^{\mathrm{E}, k^{*}}-\mathbf{X}^{\mathrm{E}, k-1^{*}}\right\| \leq \sigma_{2}
$$

\section{CASE STUdy}

The T118D30 system in Fig. 2, which has the IEEE 118bus TS connected with thirty 33-bus DSs, is used to present the computation efficiency and restoration performance of the proposed distributed risk-limiting load restoration method. Detailed data about the system can be found in Appendix B. The confidence level $\beta$ is set as $85 \%$. The uncertain data of wind power output and load amount follow the beta distribution and normal distribution with $5 \%$ forecast error, respectively with $1 \mathrm{~s}$ resolution. The parameter $\rho$ is set as 10 and the residuals $\sigma_{1}$ and $\sigma_{2}$ are set as 0.001 and 0.01 , respectively. All case studies were conducted using Gurobi V7.5.1 on a computer with the Intel(R) Core(TM) i5-2400 CPU and 4 GB RAM.

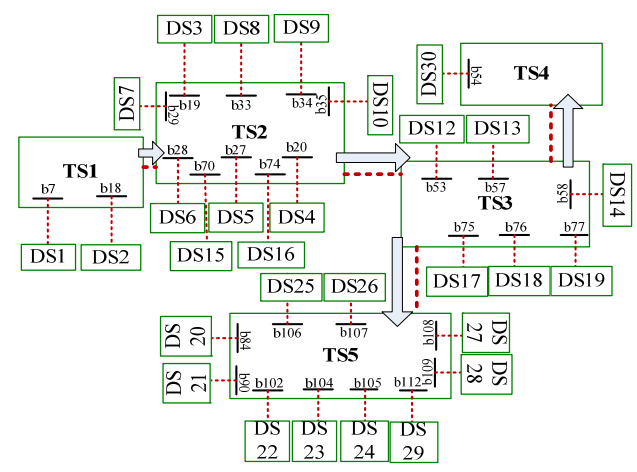

Fig. 2. The construction of the T118D30 system

\section{A. Computation performance of distributed risk-limiting method}

Compared with the conventional centralized risk-limiting method in [17], the distributed method solves simpler mathematical models. As for the optimization model, the centralized method solves a MILP problem, while the proposed distributed method solves QP problems and obtains the results of binary variables by projection functions. Regarding the model scale, the centralized model comprises the whole T118D30 system with 1180 nodes and 1407 lines, while the proposed method independently models 35 subsystems, the largest one comprising 34 nodes and 46 lines. Besides, the CVaR based risk constraints introduce 600 mixinteger constraints in the centralized method, while those constraints are all linear in the proposed method. Overall, the distributed risk-limiting method simplifies the process by transforming the model into a simpler programming type and reducing the number of variables and constraints.

As shown in Fig. 3, the distributed risk-limiting method obtains the result by iterations. It solves QP problems of 35 subsystems in parallel after 71 iterations, and the result is 376.72 MW which is close to $383.45 \mathrm{MW}$ obtained by the centralized method. Although a slightly lower load is restored, the risk is substantially reduced, as demonstrated in the next subsection. During the iteration process, each subsystem makes decisions independently and adjusts its boundary 
variables according to the result of each iteration. As shown in Fig. 3 (b) and (c), the primal and dual residuals gradually decrease and finally converge. The convergence of the primal residuals guarantees the feasibility of the obtained restoration scheme.
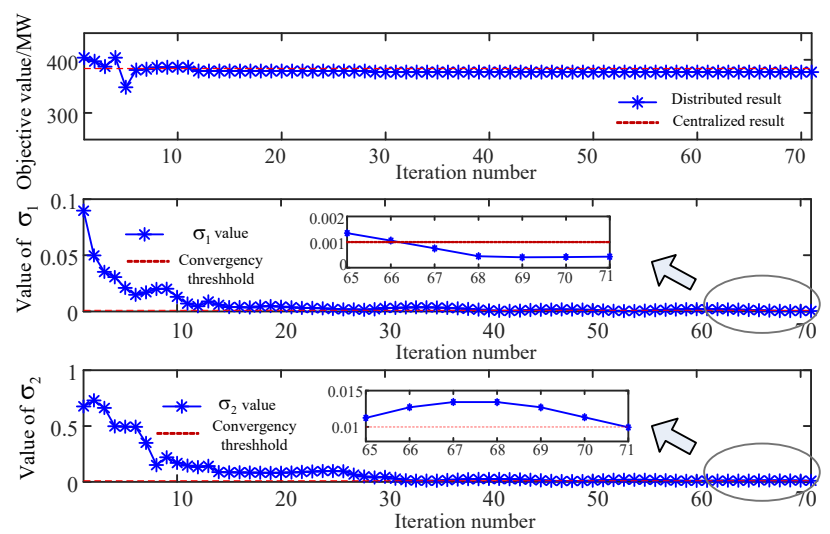

Fig. 3. The result of distributed and centralized method (a), the iteration process of the maximal primal residual (b) and dual residual (c)

The proposed distributed risk-limiting method maintains the flexible uncertainty management characteristic of the CVaR method. Fig. 4 shows the load pickup amount with different confidence levels. Under the same risk bound, the increase of the confidence level leads to a more conservative load recovery strategy with less load pickup, which is expected.

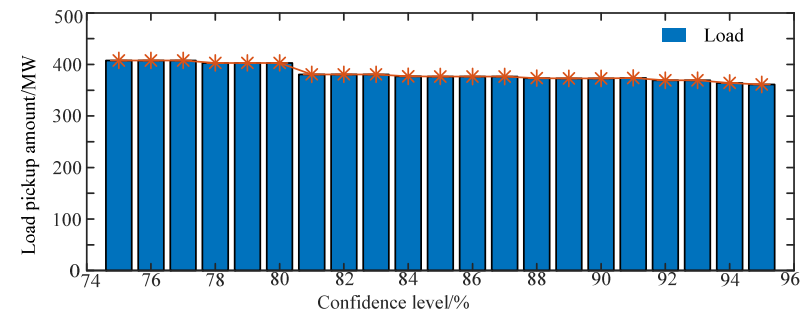

Fig. 4. Load pickup amount of different confidence level

Fig. 5 shows the load pickup amount with different forecast errors of wind power output. Under the same confidence level, the load recovery amount decreases with the increase of forecast errors. A larger forecast error means that the uncertain condition is more serious with a larger system risk value. The proposed method identifies the uncertain condition by the CVaR based risk index. Consequently, less wind power generation is used when the forecast error is larger, which leads to smaller load pickup amount.

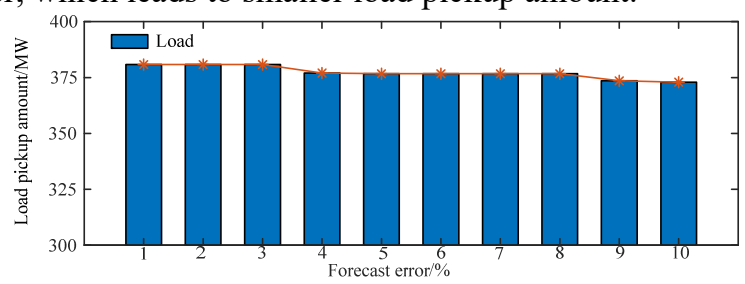

Fig. 5. Impact of the wind power generation uncertainty

\section{B. Load restoration performance with the distributed risk- limiting scheme}

The multi-step load restoration processes obtained by three methods are listed in Table I. The three methods are: 1) The proposed Distributed Risk Limiting method (DRL), 2) The Centralized Risk Limiting method (CRL) [17], 3) The conventional method that models DSs as load blocks with Risk limiting consideration (Conv-RL) [2]. The confidence level is $85 \%$.

TABLE I

RESTORATION EFFICIENCY COMPARISON OF DIFFERENT METHODS

\begin{tabular}{|c|c|c|c|c|c|c|c|c|c|c|}
\hline \multicolumn{2}{|c|}{ Steps } & S1 & S2 & S3 & S4 & S5 & S6 & S7 & S8 & S9 \\
\hline \multirow{3}{*}{$\begin{array}{l}\text { Step time } \\
\text { (min) }\end{array}$} & DRL & 10 & 10 & 10 & 9.90 & 9.87 & 9.48 & 8.56 & 9.64 & 9.63 \\
\hline & CRL & 10 & 10 & 10 & 10 & 8.17 & 10 & 10 & 9.53 & 7.55 \\
\hline & Conv-RL & 10 & 10 & 10 & 10 & 1 & 1 & 1 & 1 & 1 \\
\hline \multirow{3}{*}{$\begin{array}{c}\text { Load } \\
\text { recovery }(\%)\end{array}$} & DRL & 29.37 & 69.7 & 85.23 & 89.91 & 91.40 & 94.12 & 98.19 & 98.47 & 100 \\
\hline & CRL & 29.89 & 72.48 & 86.77 & 89.36 & 90.72 & 95.48 & 98.82 & 99.14 & 100 \\
\hline & Conv-RL & 23.85 & 54.15 & 65.69 & 72.23 & 1 & 1 & 1 & 1 & 1 \\
\hline \multirow{2}{*}{$\begin{array}{c}\text { Computation } \\
\text { time }(\mathrm{s})\end{array}$} & DRL & 5.82 & 2.87 & 6.18 & 5.28 & 3.27 & 1.86 & 2.35 & 1.00 & 1.13 \\
\hline & CRL & 4.51 & 4.76 & 3.81 & 3.25 & 3.12 & 2.56 & 3.17 & 3.35 & 2.03 \\
\hline \multirow{2}{*}{$\begin{array}{c}\text { Risk index } \\
\text { CVaR }\end{array}$} & DRL & 5.21 & 6.06 & 4.26 & 4.74 & 3.82 & 3.03 & 4.15 & 5.37 & 4.88 \\
\hline & CRL & \multicolumn{9}{|c|}{ The whole system risk bound: 6.47} \\
\hline
\end{tabular}

It is shown that the Conv-RL method shows the worst load restoration performance. The load recovery takes more time and cannot continue when it reaches $72.23 \%$ of the load. That is because each DS is treated as a load block corresponding to one binary variable, thus reducing the feasible region of the load restoration optimization. Moreover, picking up the remaining load blocks will cause violation of risk constraints due to their sizes and uncertainty. On the contrary, both DRL and CRL methods that consider the detailed characteristic of DSs, achieve $90 \%$ load recovery within $50 \mathrm{~min}$ and complete the whole process within 88 min in 9 restoration steps. Regarding risk, DRL has lower CVaR in each step than CRL with a risk bound of 6.47. This means that the distributed risklimiting load restoration strategy reduces the frequency security violation risk of the interconnected bulk system and is feasible for the whole system security, as long as the risk constraints of each subsystem are satisfied.

The computation time of both DRL and CRL decreases along the restoration process. This is because the binary variables are gradually fixed and variables and constraints are reduced in the CRL. In the DRL, both the model scale and the iteration time are reduced. The computation time of the nine steps of the DRL is $29.76 \mathrm{~s}$ which is smaller than $30.26 \mathrm{~s}$ of the CRL. The benefit of less computation time with the DRL will be more obvious when larger systems are studied or more restoration steps are needed. It should be also noted that the CRL may not be applicable in real interconnected bulk system restoration, since TSOs have limited knowledge of data at the distribution level and information exchange among TSOs is also limited. In any case, the additional time delays for collecting all information at a central location needs to be considered.

The iteration process of 9 restoration steps of the DRL method is shown in Fig. 6. Two boundary variables are demonstrated: boundary power between TSs (TS3 to TS4) and boundary power between TS and DS (TS3 to DS17). $P_{\mathrm{B}, \mathrm{TS}}, P_{\mathrm{B}}$, ${ }_{\mathrm{TS} 4}$ and $P_{\mathrm{B}, \mathrm{TS} 3}^{\mathrm{g}}$ denote the boundary power in TS3, the one in TS4 and the auxiliary variables between TS3 and TS4. $P_{\mathrm{B}, \mathrm{TD} r}, P_{\mathrm{B}, \mathrm{D}, 7}$ and $P_{\mathrm{B}, \mathrm{TD} 77}^{\mathrm{g}}$ represent the boundary power in TS3, the one in DS17 and the auxiliary variables between TS3 and DS17. CR 


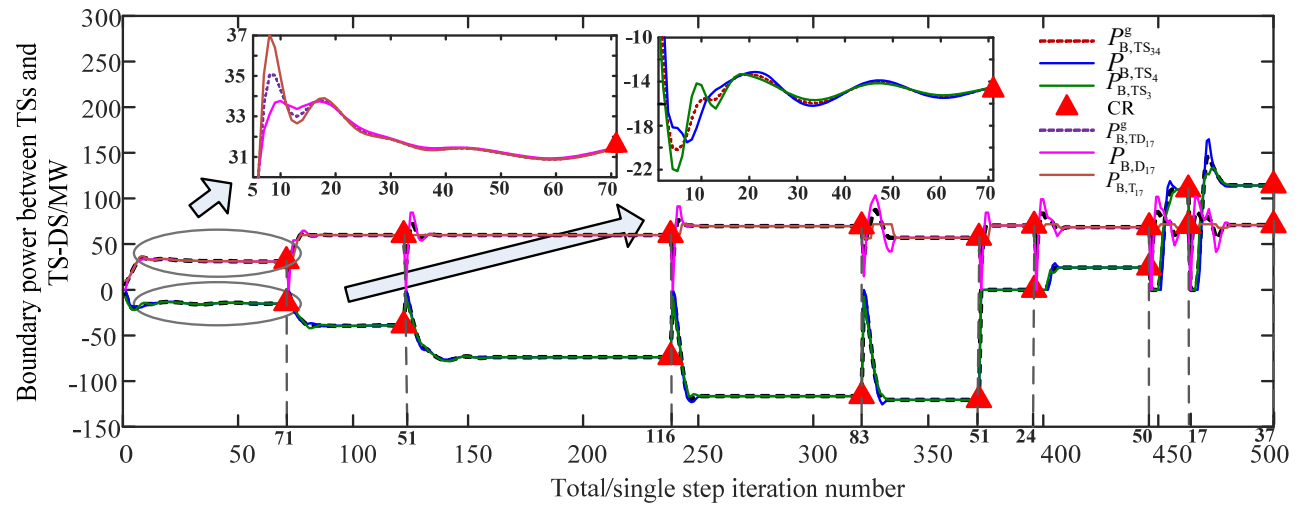

Fig. 6. Boundary powers between TS3 and TS4 and TS3 and DS17

means the final converged result of each step. As shown in Fig. 6 , the iteration times are less in later steps, since the number of binary variables is reduced as restoration continues. In each step, the boundary variables get closer to each other in this iteration process and converge to the same value. In this way, the sub-problems are independently solved, but coordinated through the alternating calculation process. It should be noted that, the boundary power between TS3 and TS4 is negative in the first five steps, 0 in the sixth step and positive in the last three steps. It means that TS4 imports power in the early stages, achieves self-support by ramping up generator outputs and utilizing wind power, and finally exports power to help TS3 to complete restoration.

The frequency condition in the distributed risk-limiting load restoration process is shown in Fig. 7. As can be seen, the largest frequency deviation of the whole interconnected system is below $0.3 \mathrm{~Hz}$ which satisfies the $0.5 \mathrm{~Hz}$ security requirement. In the load restoration process, large frequency changes normally happen when picking up loads in each step, and the fluctuation comes from the uncertainties of wind power and load amount.

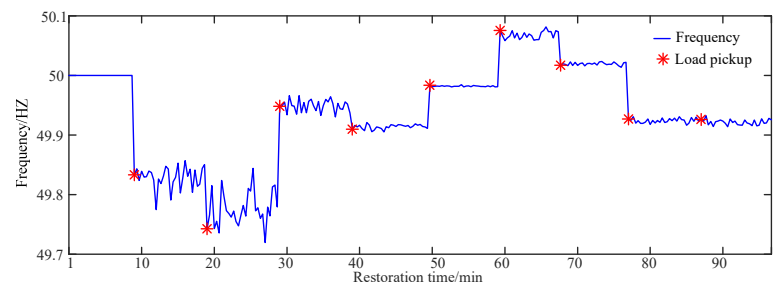

Fig. 7. Frequency condition in the whole load restoration process

\section{CONCLUSION}

This paper proposes a new distributed risk-limiting load restoration scheme for the interconnected bulk system. The distributed risk-limiting load restoration model is solved with the CVaR integrated P-ADMM algorithm. With the distributed risk-limiting scheme, independent decision-making of diffident TSOs and DSOs is achieved, and load blocks are modeled in detail. The whole system security is maintained with the distributed risk-limiting constraints. By applying the proposed algorithm, the optimal solution of the large-scale risklimiting load restoration problem is obtained by solving smallscale QP problems in parallel with projected binary variables. The case study results show the computational benefits and restoration performance of the proposed method. More specifically, the CVaR integrated P-ADMM algorithm reduces the computation complexity without loss of optimality and provides flexible uncertainty management. The distributed risklimiting load restoration scheme ensures full recovery of loads under uncertain conditions and reduces the security violation risk of the interconnected bulk system during the load restoration process.

\section{APPENDIX A}

The constraints of the TS and DS are presented as follows:

1) Constraints for the TS. Generator output limits are (A-1)(A-2) [5], [6], which means each generator is ramping up within the lower and upper generation limits. Considering the $\mathrm{N}-1$ security criterion of generator, (A-3) ensures that the required spinning reserve is provided in the restoration procedure. Each restoration step is constraint by the load pickup time (e.g. 5-10 $\mathrm{min}$ ) (A-4). Constraints (A-5)-(A-12) are linearized $\mathrm{AC}$ power flow equations for the meshed transmission network [2]. (A-5) and (A-6) determine the active and reactive power injections at each node of the $i$ th TS, (A-7) and (A-8) model KCL at the nodes, constraints (A-9) and (A-10) capture the approximate line flows from the original ones, (A-11) defines a system of inequalities capturing the polyhedral relaxation of cosine using $2 h$ hyperplanes for each line in the $i$ th TS, and (A-12) calculates the voltage of each node. The voltage constraint is (A-13). Constraints (A-14)-(A-17) are square constraints to approximate the quadratic constraint of branch apparent power limit. (A-18) is the TS boundary power limit.

$$
\begin{aligned}
& p_{\mathrm{G}_{\mathrm{TS}}, j} \geq \max \left\{p_{\mathrm{G}_{\mathrm{T}}, \mathrm{TS}_{i}, j}^{0}+r_{j} T, p_{\mathrm{G}_{\mathrm{TS}}, j, \text { min }}\right\} \forall j \in N_{\mathrm{G}_{\mathrm{TS}}} \\
& p_{\mathrm{G}, \mathrm{TS}_{i}, j} \leq \min \left\{p_{\mathrm{G}, \mathrm{TS}_{i}, j}^{0}+r_{j} T, p_{\mathrm{G}, \mathrm{TS}_{i}, j, \max }\right\} \forall j \in N_{\mathrm{G}, \mathrm{TS}_{i}} \\
& p_{\mathrm{G}, \mathrm{TS}_{i}, j} \leq \sum_{j^{e} \in N_{\mathrm{G}, \mathrm{TS}_{i}, j} j^{e} \neq j}\left(r_{j^{\prime}} T_{\max }+p_{\mathrm{G}, \mathrm{TS}_{i}, j^{e}}\right)+\sum_{j \in N_{\mathrm{RE}, \mathrm{TS}}} x_{\mathrm{RE}, \mathrm{TS} i, j}^{\mathrm{c}} p_{\mathrm{RE}, \mathrm{TS}_{i}, j} \\
& -\sum_{j \in N_{\mathrm{T}, \mathrm{TS}} \& N_{\mathrm{TD}, \mathrm{TS}}} P_{\mathrm{B}, \mathrm{TS}, j}-\sum_{j \in N_{\mathrm{TL}, \mathrm{TS}_{i}}}\left(p_{\mathrm{TL}, \mathrm{TS}}, j x_{\mathrm{TL}, \mathrm{TS} i}, j\right) \\
& T_{\min } \leq T \leq T_{\max } \\
& p_{n}=x_{\mathrm{RE}, \mathrm{TS}_{i}, n}^{\mathrm{c}} p_{\mathrm{RE}, \mathrm{TS}_{i}, n}\left(n \in N_{\mathrm{RE}, \mathrm{TS}_{i}}\right) \text { or } p_{\mathrm{G}, \mathrm{TS}}, n\left(n \in N_{\mathrm{G}, \mathrm{TS}_{i}}\right) \\
& o r-P_{\mathrm{B}, \mathrm{TS}_{i}, n}\left(n \in N_{\mathrm{T}, \mathrm{TS}}\right) o r-P_{\mathrm{B}, \mathrm{TS}_{i}, n}\left(n \in N_{\mathrm{TD}, \mathrm{TS}_{i}}\right) \\
& o r-x_{\mathrm{TL}, \mathrm{TS}, i} p_{\mathrm{TL}, \mathrm{TS}, i}\left(n \in N_{\mathrm{TL}_{\mathrm{TS}}}\right)+0 \\
& q_{n}=-x_{\mathrm{TL}, \mathrm{TS}_{i}, n} q_{\mathrm{TL}, \mathrm{TS}_{i}, n}\left(n \in N_{\mathrm{TL}^{\mathrm{TS}} \mathrm{TS}_{i}}\right) \text { or } q_{\mathrm{G}_{\mathrm{T}} \mathrm{TS}_{i}, n}\left(n \in N_{\mathrm{G}_{\mathrm{TS}}}\right)+0(\mathrm{~A}-6) \\
& p_{n}=\sum_{\langle n, m\rangle \in L_{\mathrm{TS}_{i}}} P_{n m} \forall n \in N_{\mathrm{TS}_{i}}
\end{aligned}
$$




$$
\begin{aligned}
& q_{n}=\sum_{\langle n, m\rangle \in L_{\mathrm{TS}_{i}}} Q_{n m} \quad \forall n \in N_{\mathrm{TS}_{i}} \\
& P_{n m}=g_{n m}-g_{n m} \cos ^{*} \theta_{n m}-b_{n m}\left(\theta_{n}-\theta_{m}\right)\langle n, m\rangle \in L_{\mathrm{TS}_{i}} \\
& Q_{n m}=-b_{n m}-g_{n m}\left(\theta_{n}-\theta_{m}\right)+b_{n m} \cos ^{*} \theta_{n m}-b_{n m}\left(\delta_{n}-\delta_{m}\right) \\
& \langle n, m\rangle \in L_{\mathrm{TS}_{i}} \\
& \cos ^{*} \theta_{n m} \leq-\sin \left(v d-\theta_{\Delta}^{0}\right)\left(\theta_{n}-\theta_{m}-v d+\theta_{\Delta}^{0}\right)+\cos \left(v d-\theta_{\Delta}^{0}\right) \\
& v=1,2, \ldots, 2 h \\
& V_{n}=1+\delta_{n} \quad \delta_{n} \text { (p.u.) } \in\left(-V_{n},+\infty\right) \\
& V_{n, \text { min }}<V_{n}<V_{n, \max } \quad \forall n \in N_{\mathrm{TS}_{i}} \\
& -S_{n m, \text { max }} \leq P_{n m} \leq S_{n m, \text { max }}\langle n, m\rangle \in L_{\mathrm{TS}_{i}} \\
& -S_{n m, \text { max }} \leq Q_{n m} \leq S_{n m, \text { max }}\langle n, m\rangle \in L_{\mathrm{TS}_{i}} \\
& -\sqrt{2} S_{n m, \text { max }} \leq P_{n m}+Q_{n m} \leq \sqrt{2} S_{n m, \text { max }}\langle n, m\rangle \in L_{\mathrm{TS}_{i}} \\
& -\sqrt{2} S_{n m, \text { max }} \leq P_{n m}-Q_{n m} \leq \sqrt{2} S_{n m, \text { max }}\langle n, m\rangle \in L_{\mathrm{TS}_{i}} \\
& -P_{\mathrm{B}, j, \text { max }} \leq P_{\mathrm{B}, \mathrm{TS}_{i}, j} \leq P_{\mathrm{B}, j, \text { max }} \quad \forall j \in N_{\mathrm{TD}, \mathrm{TS}_{i}}
\end{aligned}
$$

2) Constraints for the DS. E.q. (A-19)-(A-23) are linearized DisFlow constraints for distribution radial networks [26], (A19)-(A-20) determine the active and reactive power injections of node $n$ in the $i$ th DS, (A-22)-(A-23) model KCL at the nodes in the radial network, and (A-24) calculates the voltage. Inequality (A-24) represents the voltage limit. (A-25)-(A-28) are square constraints to approximate the quadratic constraint of DS branch power loss limit. The DS boundary power limit is $(\mathrm{A}-29)$.

$$
\begin{aligned}
& p_{\mathrm{DS}, n}=-x_{\mathrm{DLLS}, n} p_{\mathrm{DL}, \mathrm{DS}, n}\left(n \in N_{\mathrm{DL}, \mathrm{DS}}\right) o r-P_{\mathrm{B}, \mathrm{DS}_{i}, n}\left(n \in N_{\mathrm{DT}, \mathrm{DS}}\right) \\
& \text { or } x_{\mathrm{DG}^{\mathrm{DDS}}, n}^{\mathrm{c}} p_{\mathrm{DG}^{\mathrm{DDS}}, n}\left(n \in N_{\mathrm{DG}_{i} \mathrm{DS}}\right)+0 \\
& q_{\mathrm{DS}, n}=-x_{\mathrm{DL}, \mathrm{DS}, n} q_{\mathrm{DL}, \mathrm{DS}, n}\left(n \in N_{\mathrm{DL}, j}\right)+0 \\
& P_{\mathrm{DS}, n_{\mathrm{in}}}=P_{\mathrm{DS}, n_{\text {out }}}-p_{\mathrm{DS}, n_{\text {in }}}\left\langle n_{\text {in }}, n_{\text {out }}\right\rangle \in L_{\mathrm{DS}} \\
& Q_{\mathrm{DS}, n_{\text {in }}}=Q_{\mathrm{DS}, n_{\text {out }}}-q_{\mathrm{DS}, n_{\text {in }}}\left\langle n_{\text {in }}, n_{\text {out }}\right\rangle \in L_{\mathrm{DS}_{i}} \\
& V_{n_{\text {in }}}=V_{n_{\text {out }}}-\left(r_{n_{\text {out }}} P_{\mathrm{DS}, n_{\text {out }}}+x_{n_{\text {out }}} Q_{\mathrm{DS}, n_{\text {out }}}\right) / V_{0} \\
& V_{n, \text { min }}<V_{n}<V_{n, \text { max }}\left(\forall n \in N_{\mathrm{DS}_{i}}\right) \\
& -V_{0} \sqrt{L_{n, \text { max }} / r_{n}} \leq P_{\mathrm{DS}, n} \leq V_{0} \sqrt{L_{n, \text { max }} / r_{n}} \\
& -V_{0} \sqrt{L_{n, \text { max }} / r_{n}} \leq Q_{\mathrm{DS}, n} \leq V_{0} \sqrt{L_{n, \max } / r_{n}} \\
& -V_{0} \sqrt{2 L_{n, \text { max }} / r_{n}} \leq P_{\mathrm{DS}, n}+Q_{\mathrm{DS}, n} \leq V_{0} \sqrt{2 L_{n, \text { max }} / r_{n}} \\
& -V_{0} \sqrt{2 L_{n, \text { max }} / r_{n}} \leq P_{\mathrm{DS}, n}-Q_{\mathrm{DS}, n} \leq V_{0} \sqrt{2 L_{n, \text { max }} / r_{n}} \\
& -P_{\mathrm{B}, n, \max } \leq P_{\mathrm{B}, \mathrm{DS}}, n \leq P_{\mathrm{B}, n, \max } \quad \forall n \in N_{\mathrm{DT}, \mathrm{DS}_{i}}
\end{aligned}
$$

where $n_{\text {in }}$ and $n_{\text {out }}$ are power injection/withdrawal nodes of a unidirectional single branch of the radial distribution network.

\section{APPENDIX B}

The T118D30 system has the IEEE-118 TS with 30 ADSs. The IEEE-118-bus system is divided into five TSs as shown in [30], and the 30 DSs are connected to the TS buses as shown in Fig. 2. Each IEEE-33 DS is with three DGs and the IEEE-118 TS is with ten wind farms. The connected bus and expected values of these uncertain sources are listed in Table B-I. The generator data are presented in Table B-II. Table BIII shows the TS boundary buses which connect the TS and 30 DSs. In Table B-III, the load amount is the total load amount of the connected IEEE-33 system. The load amount of each load block in the ADS is proportionally distributed according to the total amount in Table B-III and the initial IEEE-33 system load amount, e.g., the total amount in Table VIII is $a$, the initial DS total load amount is $b$ and the DS load amount connected to one bus is $c$, then, the used load amount is $b / a^{*} c$. All the other data are from the standard IEEE-118 system and IEEE-33 system.

Initial values of boundary variables are set as 0 , and initial values of binary variables of load pickup are obtained by a frequency security concerned evaluation as shown in Model (B-1).

$$
\begin{aligned}
& \operatorname{Max} \sum_{\mathrm{TS}_{i} \in N_{\mathrm{TS}}} \sum_{j \in N_{\mathrm{TL}, \mathrm{TS}_{i}}}\left(c_{\mathrm{TL}, \mathrm{TS} i}, j p_{\mathrm{TL}, \mathrm{TS} i}, j x_{\mathrm{TL}, \mathrm{TS} i}, j\right) \\
& +\sum_{\mathrm{DS}_{i} \in N_{\mathrm{DS}}} \sum_{j \in N_{\mathrm{DL}, \mathrm{DS}}}\left(c_{\mathrm{DL}, \mathrm{DS}}, j p_{\mathrm{DL}, \mathrm{DS}}, j x_{\mathrm{DL}_{i}, \mathrm{DS}_{i}, j}\right) \\
& \text { s.t. } \quad \Delta p_{\mathrm{TL}}+\Delta p_{\mathrm{DL}} \leq \Delta f_{\max } \sum_{\mathrm{TS}_{i} \in N_{\mathrm{TS}}} \sum_{j \in N_{\mathrm{G}, \mathrm{TS}}} \frac{s_{\mathrm{G}, \mathrm{TS}}, j}{\varepsilon_{\mathrm{G}, \mathrm{TS}}, j} \\
& \Delta p_{\mathrm{TL}}+\Delta p_{\mathrm{DL}} \\
& =\sum_{\mathrm{TS}_{i} \in N_{\mathrm{TS}}} \sum_{j \in N_{\mathrm{TL}}, \mathrm{TS}_{i}}\left(x_{\mathrm{TL}, \mathrm{TS}_{i}, j}-x_{\mathrm{TL}, \mathrm{TS}_{i}, j}^{0}\right) p_{\mathrm{TL}_{\mathrm{TS}}, j} \\
& +\sum_{\mathrm{DS}_{i} \in N_{\mathrm{DS}}} \sum_{j \in N_{\mathrm{DL}, \mathrm{DS}}}\left(x_{\mathrm{DL}_{i} \mathrm{DS} i, j}-x_{\mathrm{DL}, \mathrm{DS}}^{0}, j\right) p_{\mathrm{DL}, \mathrm{DS}}, j
\end{aligned}
$$

TABLE B-I

DG OUTPUT DATA IN DisTRIBUTION SYSTEM

\begin{tabular}{cccccc}
\hline \hline DS bus & P(MW) & TS bus & P(MW) & TS bus & P(MW) \\
\hline 6 & 100 & 6 & 20 & 70 & 20 \\
\hdashline 18 & 70 & 18 & 15 & 77 & 20 \\
\hline 33 & 30 & 32 & 25 & 99 & 25 \\
\hline & & 42 & 22 & 113 & 20 \\
\hline- & 56 & 15 & 116 & 22 \\
\hline \hline
\end{tabular}

TABLE B-II

GENERATOR DATA

\begin{tabular}{ccccccc}
\hline \hline Bus & $\mathrm{P}$ & $\mathrm{Q}_{\max }$ & $\mathrm{Q}_{\min }$ & $\mathrm{P}_{\max }$ & $\mathrm{P}_{\min }$ & $r(\mathrm{MW} / \mathrm{h})$ \\
\hline 10 & 285.52 & 200 & -147 & 550 & 0 & 200 \\
\hline 12 & 19.24 & 120 & -35 & 185 & 0 & 90 \\
\hline 25 & 154.44 & 140 & -47 & 320 & 0 & 180 \\
\hline 26 & 111.56 & 1000 & -1000 & 414 & 0 & 180 \\
\hline 31 & 0 & 300 & -300 & 107 & 0 & 60 \\
\hline 46 & 112.78 & 100 & -100 & 119 & 0 & 60 \\
\hline 49 & 215.87 & 210 & -85 & 304 & 0 & 180 \\
\hline 54 & 41.62 & 300 & -300 & 148 & 0 & 60 \\
\hline 59 & 188.43 & 180 & -60 & 255 & 0 & 90 \\
\hline 61 & 185.15 & 300 & -100 & 260 & 0 & 90 \\
\hline 65 & 202.59 & 200 & -67 & 491 & 0 & 200 \\
\hline 66 & 202.83 & 200 & -67 & 492 & 0 & 200 \\
\hline 69 & 257.03 & 999 & -300 & 805.2 & 0 & 420 \\
\hline 80 & 140.23 & 280 & -165 & 577 & 0 & 200 \\
\hline 87 & 0 & 1000 & -100 & 104 & 0 & 60 \\
\hline 89 & 309.12 & 300 & -210 & 707 & 0 & 400 \\
\hline 100 & 243.48 & 155 & -50 & 352 & 0 & 180 \\
\hline 103 & 0 & 40 & -15 & 140 & 0 & 60 \\
\hline 111 & 0 & 1000 & -100 & 136 & 0 & 60 \\
\hline \hline \multicolumn{6}{c}{} & 0
\end{tabular}


TABLE B-III

TS BOUNDARY BUSES AND THE CONNECTED DS TOTAL LOAD AMOUNT

TS Bus [7 181920272829333435395357587074757677 $8490102104105106107108109112118]$

Load amount [49604548 71 47 34 435933 3743324266 68 61 41 (MW) $163553831435032486853]$

\section{REFERENCES}

[1] L. H. Fink, K. L. Liou, and C. C. Liu, "From generic restoration actions to specific restoration strategies," IEEE Trans. Power Syst., vol. 10, no. 2, pp. 745-751, 1995.

[2] J. Zhao, H. Wang, Y. Liu, R. Azizipanah-Abarghooee and V. Terzija, "Utility-oriented online load restoration considering wind power penetration," IEEE Trans. Sustain. Energy, vol. 10, no. 2, pp. 706-717, 2019.

[3] H. Qu and Y. Liu, "Maximizing restorable load amount for specific substation during system restoration," Int. J. Elect. Power Energy Syst., vol. 43, no. 1, pp. 1213-1220, 2012.

[4] D. R. Medina, E. Rappold, O. Sanchez, X. Luo, S. R. R. Rodriguez, D. Wu and J. N. Jiang, "Fast assessment of frequency response of cold load pickup in power system restoration," IEEE Trans. Power Syst, vol. 31, no. 4, pp. 3249-3256, 2016.

[5] Z. Qin, Y. Hou, C. C. Liu, S. Liu and W. Sun, "Coordinating generation and load pickup during load restoration with discrete load increments and reserve constraints," IET Gener. Transm. Distrib., vol. 9, no. 15, pp. 2437-2446, 2015.

[6] A. Gholami, W. Sun, Qun Zhou, Q. P. Zheng and Y. Hou, "Incorporating wind energy in power system restoration planning," IEEE Trans. Smart Grid, vol.10, no. 1, pp. 16-28, 2019.

[7] A. Golshani, W. Sun, Q. Zhou, Q. P. Zheng, J. Wong and F. Qiu, "Coordination of wind farm and pumped-storage hydro for a self-healing power grid," IEEE Trans. Sustain. energy, vol. 9, no. 4, pp. 1910-1920, 2018.

[8] Continental Europe operation handbook. Policy 5: emergency operations' 2017, [Online]. Available:

https://docstore.entsoe.eu/Documents/Publications/SOC/Continental_Eu rope/oh/170926_Policy_5_ver3_1_43_RGCE_Plenary_approved.pdf

[9] Z. Li, Q. Guo, H. Sun, and J. Wang, "Coordinated economic dispatch of coupled transmission and distribution systems using heterogeneous decomposition," IEEE Trans. Power Syst., vol. 31, no. 6, pp. 4817-1830, 2016.

[10] Zhigang Li, M. Shahidepour, W. Wu, B. Zeng, B. Zhang and W. Zheng, "Decentralized multiarea robust generation unit and tie-line scheduling under wind power uncertainty" IEEE Trans. Sustain. energy, vol. 30, no. 4, pp. 1925-1936, 2015.

[11] D. K. Molzahn, F. Dorfler, H. Sandberg, S. H. Low, S. Chakrabati, R. Baldick and J. Lavaei, "A survey of distributed optimization and control algorithms for electric power systems," IEEE Trans. Smart Grid, vol. 8, no. 6, pp. 2941-2962, 2017.

[12] S. Boyd, N. Parikh, E. Chu, B. Peleato, and J. Eckstein, "Distributed optimization and statistical learning via the alternating direction method of multipliers," Foundations and Trends in Machine Learning, vol. 3, no. 1, pp. 1-122, 2010.

[13] W. Zheng, W. Wu, B. Zhang, H. Sun, and Y. Liu, "A fully distributed reactive power optimization and control method for active distribution networks," IEEE Trans. Smart Grid, vol. 7, no. 2, pp. 1021-1033, 2016.

[14] Y. Guo, H. Gao, H. Xing, Q. Wu and Z. Lin, "Decentralized coordinated voltage control for VSC-HVDC connected wind farms based on ADMM", IEEE Trans. Sustain. energy, in press.

[15] Y. Zhao, Z. Lin, Y. Ding, Y. Liu, L. Sun and Y. Yan, "A model predictive control based generator start-up optimization strategy for restoration with microgrids as black-start resources" IEEE Trans. Power Syst., in press.

[16] P. P. Varaiya, F. F. Wu and J. W. Bialek, "Smart operation of smart grid: risk limiting dispatch," Proc. IEEE, vol. 99, no. 1, pp. 40-57, Jan. 2011.

[17] C. Peng, Y. Hou, N. Yu and W. Wang, "Risk-limiting unit commitment in smart grid with intelligent periphery," IEEE Trans. Power Syst., vol. 32, no. 6, pp. 4696-4707, 2017.

[18] C. Peng, Y. Hou, N. Yu, J. Yan, S. Lei and W. Wang, "Multiperiod risklimiting dispatch in power systems with renewables integration," IEEE Trans. Indus. Inform., vol. 13, no. 4, pp. 1843-1854, 2017.
[19] Z. Wang, C. Shen, Y. Xu, F. Liu, X. Wu and C. C. Liu, "Risk-limiting load restoration for resilience enhancement with intermittent energy resources," IEEE Trans. Smart Grid, vol.10, no. 3, pp. 2507-2522, 2019.

[20] H. Wu, M. Shahidehpour, A. Alabdulwahab and A. Abusorrah, "A game theoretic approach to risk-based optimal bidding strategies for electric vehicle aggregators in electricity markets with variable wind energy resources," IEEE Trans. Sustain. energy, vol. 7, no. 1, pp. 374-385, 2016.

[21] A. Rabiee, A. Soroudi and A. Keane, "Risk-averse preventive voltage control of AC/DC power systems including wind power generation," IEEE Trans. Sustain. energy, vol. 6, no. 4, pp. 1494-1505, 2015.

[22] R. T. Rockafellar and S. Uryasev, "Optimization of conditional valueatrisk," J. risk, vol. 2, pp. 21-42, 1999.

[23] G. C. Pflug, "Some remarks on the value-at-risk and the conditional value-at-risk", Probabilistic constrained optimization. Springer 2000.

[24] R. Takapoui, N. Moehle, S. Boyd and A. Bemporad, "A simple effective heuristic for embedded mixed-integer quadratic programming," in Proc. American Control Conf., Boston, Massachusetts, pp. 5619-5625, 2016.

[25] A. Alborz and M. C. Rotkowitz, "Improving ADMM-based optimization of mixed integer objectives," in Annual Conf. on Information Sciences and Systems, Baltimore, MD, USA, May 2017.

[26] H. Yeh, D. F. Gayme and S. H. Low, "Adaptive VAR control for distribution circuit with photovoltaic generators," IEEE Trans. Power Syst., vol. 27, no. 3, pp 1656-1663, 2012.

[27] (Jun. 2018) PJM Manual 36: System Restoration. [online]. Available: https://www.pjm.com/ /media/documents/manuals/m36.ashx

[28] M. M. Adibi, J. N. Borkoski, R. J. Kafka and T. L. Volkmann, "Frequency response of prime movers during restoration," IEEE Trans. Power Syst, vol.14, no.2, pp. 751-756, May. 1999.

[29] P. Krokhmal, J. Palmquist and S. Uryasev, "Portfolio optimization with conditional value-at-risk objective and constraints", J. risk, vol. 4, pp. $43-68,2002$.

[30] Z. Lin, F. Wen, C. Chung, K. Pong and H. Zhou, "Division algorithm and interconnection strategy of restoration subsystems based on complex network theory", IET Gener., Trans. \& Distr. Vol. 5, no. 6, pp. 674-683, 2011.

Jin Zhao (S'17) received the B.Sc. degree in electrical engineering in 2015

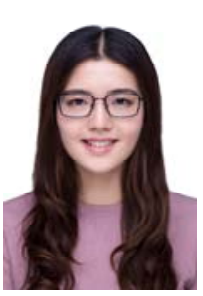
from Shandong University, Jinan, China, where she is working toward the Ph.D. degree in electrical engineering. She was a research assistant at The University of Hong Kong, China, from Sep. 2018 to Dec. 2018. From 2018 to 2019, she was a visiting student at the Department of Electrical Engineering, Technical University of Denmark. Her research interests include power system resilience, restoration, renewable energy integration, and power system optimization.

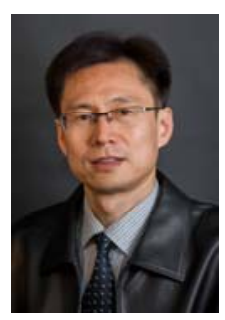

Hongtao Wang (M'04-SM'15) received the B.Sc. and M.Sc. degrees in electrical engineering from Shandong Polytechnic University, Jinan, China, in 1995 and 1998 respectively, and the Ph.D. degree in electrical engineering from Shandong University, Jinan, in 2005.

He was a Postdoctoral Researcher with the Department of Computer Science, Shandong University. From 2005 to 2010, he was an Associate Professor with the School of Electrical Engineering, Shandong University, where he has been a Professor since 2010. His research interests include power system analysis and control and smart grids.

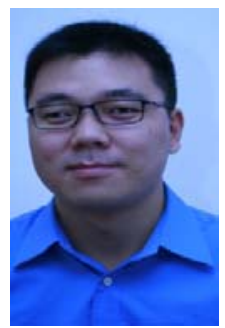

Qiuwei Wu (M'08-SM'15) obtained the $\mathrm{PhD}$ degree in Power System Engineering from Nanyang Technological University, Singapore, in 2009.

He was a senior R\&D engineer with VESTAS Technology R\&D Singapore Pte Ltd from Mar. 2008 to Oct. 2009. He has been working at Department of Electrical Engineering, Technical University of Denmark (DTU) since Nov. 2009 (PostDoc Nov. 2009-Oct. 2010, Assistant Professor Nov. 2010-Aug. 2013, Associate Professor since Sept. 2013). He was a visiting scholar at Department of Industrial Engineering \& Operations Research (IEOR), University of California, Berkeley, from Feb. 2012 to May 2012 funded by the Danish Agency for Science, Technology and Innovation (DASTI), Denmark. He was a visiting professor named by Y. Xue, an Academician of Chinese Academy of Engineering, at Shandong University, China, 
from Nov. 2015 to Oct. 2017. He was a visiting scholar at the Harvard China Project, School of Engineering and Applied Sciences, Harvard University from Nov. 2017 - Oct. 2018 funded by the Otto Mønsted Fond. His research interests are operation and control of power systems with high penetration of renewables, including wind power modelling and control, active distribution networks, and operation of integrated energy systems. He is an Editor of IEEE Transactions on Smart Grid and IEEE Power Engineering Letters. He is the deputy editor-in-chief of International Journal of Electrical Power and Energy Systems. He is a subject editor of IET Generation, Transmission \& Distribution, and the regional editor for Europe of IET Renewable Power Generation.

Nikos D. Hatziargyriou is full professor in Power Systems at the Electrical

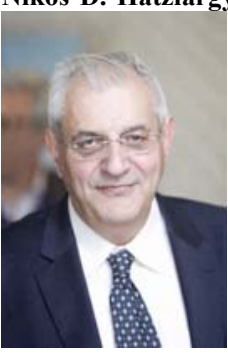
and Computer Engineering School of the National Technical University of Athens. From 2015 to 2019 he was Chair and until 2018, CEO of the Hellenic Distribution Network Operator (HEDNO). From 2007 until 2012 he was executive Vice-Chair and Deputy CEO of the Public Power Corporation (PPC), responsible for the Transmission and Distribution Divisions. He is Fellow Member of IEEE, past Chair of the Power System Dynamic Performance Committee (PSDPC) and currently Editor in Chief of the IEEE Trans on Power Systems. He is honorary member of CIGRE and past Chair of CIGRE SC C6 "Distribution Systems and Distributed Generation". He was chair and currently vice-chair of the EU Technology and Innovation Platform on Smart Networks for Energy Transition (ETIP-SNET). He has participated in more than $60 \mathrm{RD} \& \mathrm{D}$ projects funded by the EU Commission, electric utilities and manufacturers. He is author of the book "Microgrids: Architectures and Control" and of more than 250 journal publications and 500 conference proceedings papers. He is included in the 2016, 2017 and 2019 Thomson Reuters lists of the top $1 \%$ most cited researchers.

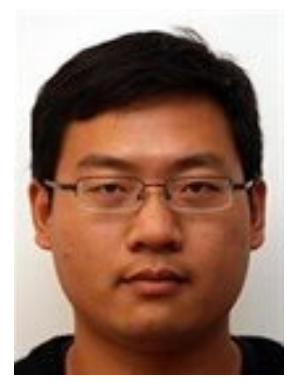

Feifan Shen received the B.Eng. degree in Electrical Engineering and Automation from Hohai University, Nanjing, China, in 2015, and the M.Eng. degree from the Department of Electrical Engineering, Wuhan University, Wuhan, China, in 2017. He is currently working toward the joint Ph.D. degree of the Department of Electrical Engineering, Centre of Electric Power and Energy, Technical University of Denmark, Kongens Lyngby, Denmark, and the School of Electrical and Electronic Engineering, Nanyang Technological University, Singapore. His research interests include congestion management and self-healing of distribution networks. 